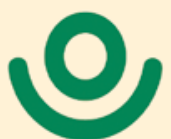

DEUTSCHE GESELLSCHAFT

FÜR KINDER- UND JUGENDMEDIZIN e.V.

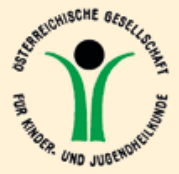

\section{ÖSTERREICHISCHE GESELLSCHAFT FÜR KINDER- UND JUGENDHEILKUNDE}

Monatsschr Kinderheilkd 2009 • 157:706-722

DOI 10.1007/s00112-009-2035-5

๑) Springer Medizin Verlag 2009

\section{Redaktion}

Prof. Dr. M. Weiß, Köln

Prof. Dr. R. Kerbl, Leoben

\section{Inhalt}

\begin{tabular}{ll}
\hline Einladung zur Mitgliederversammlung der DGKJ & 706 \\
Tagesordnung der Mitgliederversammlung 2009 & 706 \\
Anlagen zu den Satzungsänderungen & 707 \\
Vorläufiges Programm der 105. Jahresversammlung der DGKJ & 709 \\
Benefizkonzert & 717 \\
Laufende Datenerhebungen im ESPED-System & 718 \\
Personalia & 719 \\
Kongresskalender & 720 \\
Geburtstage & 721 \\
Anträge und Formulare & 722
\end{tabular}

\section{Einladung zur Mitglieder- versammlung der DGKJ}

\author{
anlässlich der 105. Jahrestagung am 05.09.2009
}

\section{Sehr geehrte Mitglieder der Deutschen Gesell- schaft für Kinder- und Jugendmedizin, liebe Kolleginnen und Kollegen,}

am 5. September 2009 um 17:30 Uhr findet die diesjährige Mitgliederversammlung unserer $\mathrm{Ge}$ sellschaft in Mannheim statt. Ich lade Sie alle hierzu sehr herzlich ein und freue mich, wenn Sie sowohl an unserer 105. Jahrestagung in Mannheim als auch an der Mitgliederversammlung teilnehmen.

Die Tagesordnung verspricht eine spannende Sitzung: Mit der Wahl des Vizepräsidenten steht ein wichtiger Punkt auf der Agenda, denn der Gewählte wird 2010 und 2011 die Aufgaben des Vizepräsidenten wahrnehmen und 2012 automatisch das Amt des Präsidenten übernehmen. Seien Sie dabei, wenn es um die Zukunft unserer Fachgesellschaft geht!

Mit freundlichen Grüßen Ihr

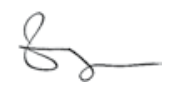

Prof. Dr. med. Fred Zepp (Präsident)

\section{Tagesordnung der Mitgliederversammlung 2009}

TOP 1: $\quad$ Festsetzung der Tagesordnung

TOP 2: Genehmigung des Protokolls der Mitgliederversammlung der 104. Jahrestagung vom 13. September 2008

TOP 3: Bericht des Präsidenten

TOP 4: Bericht des Schatzmeisters

TOP 5: $\quad$ Bericht der Kassenprüfer 2009

TOP 6: $\quad$ Entlastung des Vorstandes

TOP 7: $\quad$ Festsetzung der Jahresbeiträge 2010

TOP 8: Wahl von 2 Kassenprüfern für 2010

TOP 9: Wahl des Vizepräsidenten 2010-11 (ab 2012 Präsidenten)

TOP 10: Satzungsänderung im Hinblick auf die Zusammensetzung des Vorstandes, betr. den Sprecher des Konvents für fachliche Zusammenarbeit, und die Aufgabe der Geschäftsführung § 9 Der Vorstand § 10 Der geschäftsführende Vorstand $\S 11$ Wahl des Vorstandes

TOP 11: Satzungsänderungen aufgrund neuer Anforderungen des Bundesministeriums für Finanzen und Beanstandungen des Finanzamtes für Körperschaften I Berlin

§ 1 Ziele und Zweck der Gesellschaft

$\S 2$ Erfüllung der Aufgaben

$\S 9$ Der Vorstand

$\S 14$ Allgemeine Regelungen

$\S 15$ Allgemeine Regelungen

$\S 16$ Allgemeine Regelungen

TOP 12: DGKJ-Mitgliederbefragung: Ergebnisse

TOP 13: Wahl des Kongresspräsidenten 2013

TOP 14: Zukünftige Entwicklung der DGKJ-Jahrestagung

TOP 15: Organisationsstruktur des Vorstandes und der Geschäftsstelle der DGKJ

TOP 16: Aufgaben der DGKJ als wissenschaftliche Fachgesellschaft in den gesundheitspolitischen Entscheidungsprozessen

TOP 17: Wahl von Ehrenmitgliedern und korrespondierenden Mitgliedern

TOP 18: Berichte der Kongresspräsidenten 2010 und 2011

TOP 19: Bericht aus dem Konvent für fachliche Zusammenarbeit

TOP 20: Bericht aus dem Hochschullehrerkonvent

TOP 21: Berichte der DGKJ-Kommissionen

TOP 22: Verschiedenes 


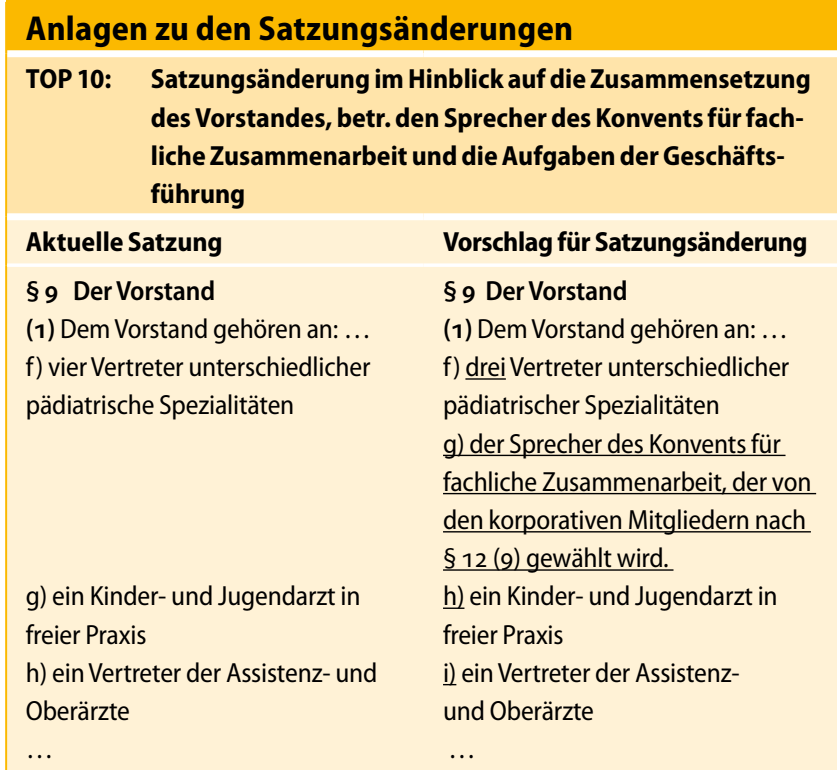

\section{(Forts.)}

(2) Die Mitglieder a) - h) sind stimmberechtigt; die kooptierten Vorstandsmitglieder i) - $\mathrm{n}$ ) besitzen Rede, aber kein Stimmrecht.

(4) Der Vorstand führt die Geschäfte der Gesellschaft, soweit sie nicht anderen Organen zugewiesen sind. Er kann der Mitglieder-versammlung die Bestellung eines Geschäftsführers vorschlagen.

\$10 Der

(1) Der geschäftsführende Vorstand setzt sich aus dem Präsidenten, dem Vizepräsidenten, dem Schatzmeister und einem in eigener Wahl ermittelten Vorstandsmitglied aus § $9, \mathrm{f})$ zusammen.

\section{\$ 11 Wahl des Vorstandes}

(1) Die Mitgliederversammlung wählt die Mitglieder des Vorstandes nach § 9, Abs. 1 Buchstaben b) bis h).

(3) Die Wahl des Vorstandsmitgliedes aus $\S 9$, f für den geschäftsführenden Vorstand erfolgt mit einfacher Mehrheit.
(2) Die Mitglieder a) - i) sind stimmberechtigt; die kooptierten Vorstandsmitglieder j) - o) besitzen Rede- aber kein Stimmrecht

(4) Der Vorstand führt die Geschäfte der Gesellschaft, soweit sie nicht anderen Organen zugewiesen sind. Er kann der Mitgliederversammlung die Bestellung eines Geschäftsführers vorschlagen. Die Geschäftsführung unterstützt den Vorstand bei der Erfüllung seiner Aufgaben. Sie ist kein Organ der Gesellschaft.

\section{§ 10 Der geschäftsführende} Vorstand

(1) Der geschäftsführende Vorstand setzt sich aus dem Präsidenten, dem Vizepräsidenten, dem Schatzmeister und einem in eigener Wahl ermittelten Vorstandsmitglied aus $\S 9$, fund g) zusammen.

\section{§ 11 Wahl des Vorstandes}

(1) Die Mitgliederversammlung wählt die Mitglieder des Vorstandes nach $\S 9$, Abs. 1 Buchstaben b) bis f), h) und i).

\section{(3) Die Wahl des}

Vorstandsmitgliedes aus $\S 9$, $f$ und g für den geschäftsführenden Vorstand erfolgt mit einfacher Mehrheit.

\section{Exklusiv für Abonnenten und Gesellschaftsmitglieder :}

\section{Nutzen Sie das Online-Archiv von Monatsschrift Kinderheilkunde} Ihre Vorteile:

- Komfortable und schnelle Recherche nach Themen, Autoren, Suchbegriffen

— Ob unterwegs oder am eigenen PC: Zugriff überall und jederzeit

_ Online First: Lesen Sie die aktuellsten Beiträge schon vor Erscheinen des gedruckten Heftes online

\section{Registrieren Sie sich jetzt unter} www.MonatsschriftKinderheilkunde.de

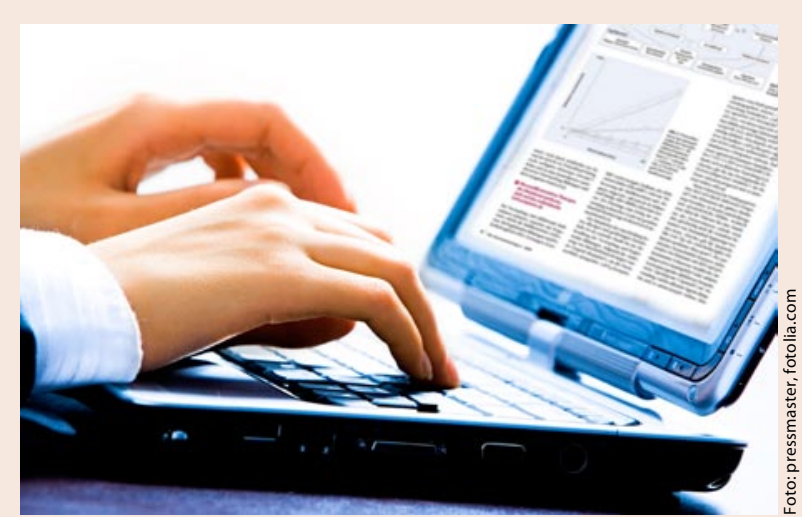

\section{Dieser Online-Service steht auch Mitgliedern der folgenden Fachgesellschaften zur Verfügung:}




\section{Anlagen zu den Satzungsänderungen}

TOP 11: Satzungsänderungen aufgrund neuer Anforderungen des Bundesministeriums für Finanzen und Beanstandungen des Finanzamtes für Körperschaften I Berlin

\section{Aktuelle Satzung}

§1Ziele und ZweckderGesellschaft

(1) Die Deutsche Gesellschaft für

Kinder- und Jugendmedizin e.V., Sitz

Berlin, verfolgt ausschließlich und

unmittelbar gemeinnützige Zwecke

im Sinne der Gemeinnützigkeitsver-

ordnung vom 24. Dezember 1953

und der Abgabenordnung 1977

$\S 52.2$ Abs. 2 in der Fassung vom

16.03.1976.

(2) Ihre Zwecke sind:

a) die wissenschaftlichen und fachlichen Belange der Kinder- und Jugendmedizin sowie die Belange der Kinder- und Jugendärzte zu fördern und den Gemeinsinn unter den Mitgliedern zu pflegen,

b) die wissenschaftlichen Grundlagen für die bestmögliche kinderärztliche Versorgung der Bevölkerung zu erarbeiten,

c) die internationale Zusammenarbeit auf dem Gebiet der Kinderund Jugendmedizin zu pflegen, d) die Integration pädiatrischer Subspezialitäten zu fördern.

\section{§ 2 Erfüllung der Aufgaben}

(1) Diese Aufgaben erfüllt die Deutsche Gesellschaft für Kinder- und Jugendmedizin e.V., indem sie ... e) die Ausbildung, Weiterbildung, Fortbildung und die Berufsausübung der Kinder- und Jugendärzte sowie die pädiatrische Forschung und die zuständigen Behörden, Gremien und Institutionen im In- und Ausland in allen Fragen dazu berät.

\section{Vorschlag für Satzungsänderung}

§1Ziele und Zweckder Gesellschaft (1) Die Deutsche Gesellschaft für Kinder- und Jugendmedizin e. V., Sitz Berlin, verfolgt ausschließlich, unmittelbar gemeinnützige Zwecke im Sinne der Abgabenordnung (AO) in der Bekanntmachung

vom 01.10.2002 und unter Berücksichtigung der seither ergangenen Gesetzesänderungen, insbesondere vom 10.10.2007.

(2) Die Gesellschaft verfolgt ausschließlich, unmittelbar und selbst die Förderung des öffentlichen Gesundheitswesens und der öffentlichen Gesundheitspflege ( $\$ 52$ Abs. 2 Nr. 3 AO). Hierunter fällt insbesondere,

a) die wissenschaftlichen und fachlichen Belange der Kinder- und Jugendmedizin sowie die Belange der Kinder- und Jugendärzte zu fördern und den Gemeinsinn unter den Mitgliedern zu pflegen,

b) die wissenschaftlichen Grundlagen für die bestmögliche kinderärztliche Versorgung der Bevölkerung zu erarbeiten,

c) die internationale Zusammenarbeit auf dem Gebiet der Kinderund Jugendmedizin zu pflegen, d) die Integration pädiatrischer Subspezialitäten zu fördern.

(3) Der Verein ist selbstlos tätig; er verfolgt nicht in erster Linie eigenwirtschaftliche Zwecke.

\section{§ 2 Erfüllung der Aufgaben}

(1) Diese Aufgaben erfüllt die Deutsche Gesellschaft für Kinder- und Jugendmedizin e.V., indem sie ... e) die Ausbildung, Weiterbildung, Fortbildung und die Berufsausübung der Kinder- und Jugendärzte sowie die pädiatrische Forschung unterstützt, soweit es sich hierbei um Projekte gemeinnütziger Körperschaften oder Körperschaften des öffentlichen Rechts handelt, und die zuständigen Behörden, Gremien und Institutionen anderer gemeinnütziger Körperschaften oder Körperschaften des öffentlichen Rechts im In- und Ausland in allen Fragen dazu berät.

(Forts.)

§ 9 Der Vorstand

§ 9 Der Vorstand

(7) Den Vorstandsmitgliedern a) i) werden Aufwendungen gegen Nachweis erstattet, die Ihnen in Ansehung ihres Amtes tatsächlich entstanden sind. Für Präsident, Vizepräsident und Schatzmeister kann eine pauschale Vergütung in angemessener Höhe für ihre sonstigen Aufwendungen gewährt werden.

Allgemein Regelungen ...

$\S 14$

Die Gesellschaft erstrebt keinerlei Gewinn. Das Vermögen der Gesellschaft und etwaige Überschüsse oder Zuwendungen dürfen ausschließlich für satzungsmäßige Zwecke verwendet werden.
Allgemein Regelungen ...

\section{$\$ 14$}

Die Gesellschaft erstrebt keinerlei

Gewinn. Das Vermögen der Gesellschaft und etwaige Überschüsse oder Zuwendungen dürfen nur ausschließlich für die satzungsmäßigen Zwecke verwendet werden. Die Mitglieder erhalten keine Zuwendungen aus Mitteln des Vereins.

\section{$\S 15$}

Es darf keine Person durch Verwaltungsaufgaben, die den Zwecken des Vereins fremd sind, oder durch unverhältnismäßig hohe Vergütungen begünstigt werden. Die Mitgliedschaft in der Deutschen Gesellschaft für Kinderund Jugendmedizin darf nicht zu Werbezwecken verwendet werden.

\section{$\S 16$}

Bei Auflösung des Vereins fällt das Vermögen dem Deutschen Roten Kreuz zu mit der Auflage, es ausschließlich für gemeinnützige Zwecke, insbesondere für die Gesundheitsförderung im Kindesund Jugendalter zu verwenden.
$\S 15$

Es darf keine Person durch Ausgaben, die den Zwecken des Vereins fremd sind, oder durch unverhältnismäßig hohe Vergütungen begünstigt werden. Die Mitgliedschaft in der Deutschen Gesellschaft für Kinderund Jugendmedizin darf nicht zu Werbezwecken verwendet werden.

$\S 16$

Bei Auflösung des Vereins oder bei Wegfall der steuerbegünstigten Zwecke fällt das Vermögen dem Deutschen Roten Kreuz zu mit der Auflage, es unmittelbar und ausschließlich für gemeinnützige Zwecke, insbesondere für die Gesundheitsförderung im Kindesund Jugendalter zu verwenden. 


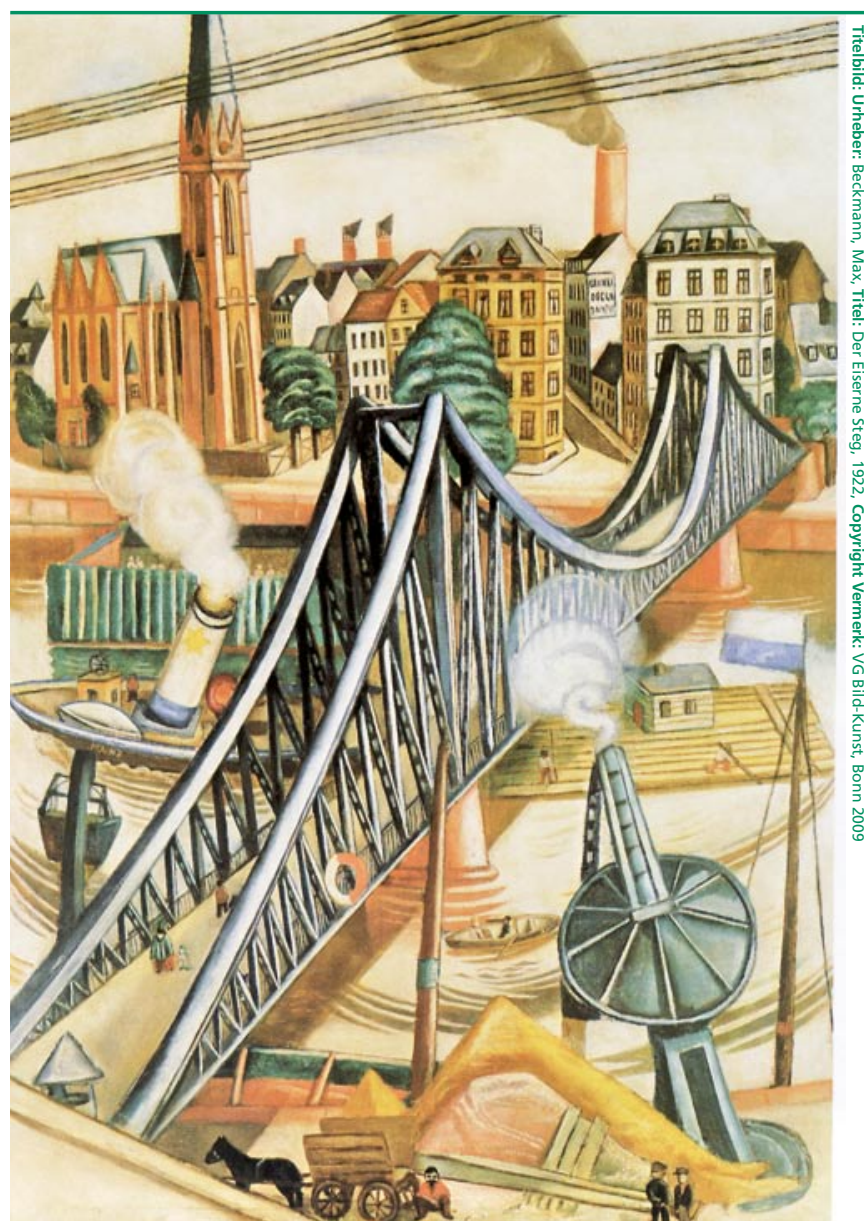

105. Jahrestagung

Deutsche Gesellschaft für Kinder- und Jugendmedizin

\section{3. - 6. September 2009 \\ Congress Center Rosengarten, Mannheim}

gemeinsam mit:

61. Jahrestagung der Deutschen Gesellschaft für Sozialpädiatrie und Jugendmedizin

47. Jahrestagung der Deutschen Gesellschaft für Kinderchirurgie

31. Tagung der Gesundheits- und KinderkrankenpflegerInnen
Donnerstag, 03.09.2009, 18:30 - 20:00 Uhr

Eröffnung der 105. wissenschaftlichen Tagung der Deutschen Gesellschaft für Kinder- und Jugendmedizin zusammen mit der

61. Jahrestagung der Deutschen Gesellschaft für Sozialpädiatrie und Jugendmedizin

47. Jahrestagung der Deutschen Gesellschaft für Kinderchirurgie

31. Tagung der Kinderkrankenschwestern und Kinderkrankenpfleger

- Begrüßung durch den Tagungspräsidenten der Deutschen Gesellschaft für Kinder- und Jugendmedizin Prof. Dr. med. HJ. Böhles

- Begrüßung durch den Tagungspräsidenten der Deutschen Gesellschaft für Kinderchirurgie Prof. Dr. med. J.L. Koltai

- Begrüßung durch den Tagungspräsidenten der Deutschen Gesellschaft für Sozialpädiatrie und Jugendmedizin

Dr. med. H. Peters

- Ansprache des Präsidenten der Deutschen Gesellschaft für Kinder- und

Jugendmedizin und

Preisverleihung

Prof. Dr. med. F. Zepp

- Festvortrag

Dr. Eckart von Hirschhausen

\section{Vorläufiges \\ Programm der DGKJ}

\section{Workshops \\ Donnerstag, 03.09.2009}

10:00 - 16:00 Uhr (PG-Kurs 1) Zertifizierte (GNPI) Fortbildungsreihe zum Erwerb der Bezeichung „NeugeborenenNotarzt"

Vorsitz: Chr. Fusch (Hamilton, Kanada)

13:00 - 17:30 Uhr (PG-Kurs 2) Station für chronisch kranke Kinder und Jugendliche - als Brücke zwischen Pädiatrie und Psychosomatik Vorsitz: G. Bürk (Herne), K. Mönkemöller (Köln)

14:30 - 17:30 Uhr (PG-Kurs 3) Unspezifische und spezifische bronchiale Provokationsmethoden

Vorsitz: S. Zielen

(Frankfurt a. M.),

J. Schulze (Frankfurt a. M.)

14:30 - 16:45 Uhr (PG-Kurs 4) Hautpflege und dermatologische Lokaltherapie im Neugeborenen- und Kleinkindesalter

Vorsitz: U. Blume-Peytavi

(Berlin)

14:30 - 17:30 Uhr (PG-Kurs 5) Onkologische Notfälle in Klinik und Praxis Vorsitz:

T. Lehrnbecher

(Frankfurt a. M.),

A. Möricke (Kiel)

14:30 - 17:30 Uhr (PG-Kurs 6) Beratung von Familien mit adipösen Kindern und Jugendlichen

Vorsitz: T. Reinehr (Datteln), Chr. Graf (Köln)

14:30 - 17:30 Uhr (PG-Kurs 7) Kohlenhydratfehlverdauung: klinische und diagnostische Fallstricke

Vorsitz: S. Koletzko (München)

14:30 - 17:30 Uhr (PG-Kurs 8) Professionelle Kommunikation in Praxen und Kliniken - Tipps und Übungen zum erfolgreichen Arbeiten Vorsitz: W. Kölfen (Mönchengladbach) 
14:30 - 16:45 Uhr (PG-Kurs 9) Leitsymptome von Immundefekten und Interpretation immunologischer Laborbefunde in Zusammenarbeit mit der AG Pädiatrische Immunologie

Vorsitz:

T. Niehues (Krefeld),

W. Mannhardt-Laakmann

(Mainz)

11:00 - 16:30 Uhr (PG-Kurs 10) GCP-Training für Prüfärzte in der Pädiatrie

Vorsitz: R. Butzer (Mainz),

R. Feneberg (Heidelberg)

14:30 - 17:30 Uhr (PG-Kurs 11)

Qualitätsmanagement in der Pädiatrie

Vorsitz: D. Ewald

(Frankfurt a. M.)

E.-M. Bitzer (Hannover)

Die Teilnehmerzahl bei den Workshops ist begrenzt. Eine Vorregistrierung wird empfohlen. (kostenpflichtig)

20:00 Uhr

Get Together

Congress Center Rosengarten

\section{Workshops}

Freitag, 04.09.2009

14:15 - 16:15 Uhr (PG-Kurs 12) B-Oxidationsstörungen - eine Herausforderung für die Pädiatrie - vom MCAD - bis zum LCHAD-Mangel

Vorsitz:

A. van Teeffelen-Heithoff

(Münster),

T. Marquardt (Münster),

M. Grotzke-Leweling

(Düsseldorf)

Plenarsitzung

Freitag, 04.09.2009

08:30 - 10:00 Uhr

Ernährung

Vorsitz:

B. Koletzko (München),

HJ. Böhles (Frankfurt a. M.)

- Proteinzufuhr und

Wachstum

E. Ziegler (lowa City, USA)

- Nutrigenomics

H. K. Biesalski (Hohenheim)

- Ernährung und Gehirnentwicklung

B. Koletzko (München)
Symposien

Freitag, 04.09.2009

10:30 - 12:00 Uhr

Ernährung und Lungenkrankheiten

Vorsitz: F. Riedel (Hamburg),

S. Zielen (Frankfurt a. M.)

- Biochemische Grundlagen von Ernährung und Funktionsstörungen der Lunge HJ. Böhles (Frankfurt a. M.)

- Richtige Ernährung und Lungenfunktion - ist dicker wirklich besser?

M. Kappler (München)

- Resolvins/Protectins:

Anti-inflammatory effects of "good" lipids in lung disease

B. Levy (Boston, USA)

10:30 - 12:00 Uhr

Ernährung und allergische Krankheiten

Vorsitz: A. Bufe (Bochum),

M.A. Rose (Frankfurt a. M.)

- Immunologie und Nahrungsmittelallergie

A. Bufe (Bochum)

- Hypoallergene Nahrung zur primären und sekundären Prävention atopischer Erkrankungen

A. von Berg (Wesel)

- Stellenwert der Probiotika in der Prävention von atopischen Erkrankungen M.A. Rose (Frankfurt a. M.)

10:30 - 12:00 Uhr

Ernährung in gastroenterologischen Krankheitsbildern Sitzung der Deutschen Gesellschaft für Pädiatrische Gastroenterologie

Vorsitz:

M. Melter (Regensburg),

S. Buderus (Bonn)

- Ernährungsempfehlungen bei akuter Pankreatitis H. Witt (München)

- Neue Empfehlungen zur Prävention und Diät der Zöliakie

K. P. Zimmer (Gießen)

- Enterale Ernährung bei Kurzdarm: Früh- und Langzeitprobleme

S. Koletzko (München)

- Ernährung bei Cholestase und Leberinsuffizienz U. Baumann (Hannover)

10:30 - 12:00 Uhr Adipositas im Kindes- und Jugendalter: Ursachen, Folgen, Behandlungsansätze Sitzung der DGKJ in Zusammenarbeit mit der AG Adipositas im Kindes- und Jugend alter und der Adipositasforschergruppe alter und der Adipositasforschergruppe
LARGE im Kompetenznetzwerk Adipositas LARGE im
des BMBF

Vorsitz: W. Kiess (Leipzig),

T. Reinehr (Datteln)
- Adipositas als polygenetische Erkrankung

J. Hebebrand (Essen)

- Fettgewebe als endokrines Organ W. Kiess (Leipzig)

- Metabolisches Syndrom und assoziierte Folgeerkrankungen der Adipositas T. Reinehr (Datteln)

- Qualitätssicherung bei Interventionen bei adipösen Kindern und Jugendlichen R. Holl (Ulm)

\section{0:30 - 12:00 Uhr}

Neonatologie und Ernährung Vorsitz:

Chr. Fusch (Hamilton, Kanada)

- Können wir die intrauterine Ernährung des Feten via Plazenta beim Frühgeborenen wirklich imitieren?

Chr. Fusch

(Hamilton, Kanada)

- Mythen und Fakten der parenteralen Ernährung G. Hellstern (Freudenstad)

- Wie sieht die ideale Milch für das Frühgeborene aus? W. A. Mihatsch (Schwäbisch-Hall)

- Ernährung der Frühgeborenen nach der Entlassung aus der Klinik

F. Jochum (Berlin)

10:30 - 12:00 Uhr

Drogenmissbrauch und Drogenentzug im Kindesalter

Vorsitz:

Chr. Möller (Hannover),

M. Kieslich (Frankfurt a. M.)

- Drogenkonsum im Kindesalter

Chr. Möller (Hannover)

- Drogenkonsumierende Patienten in der kinderärztlichen Praxis

W.-R. Horn (Gernsbach)

- Therapie und Versorgungsstrukturen

Chr. Möller (Hannover)

- Suchtprävention im Kindesalter

M. Stolle (Hamburg)

\section{Industriesymposien \\ Freitag, 04.09.2009}

12:15 - 13:45 Uhr

Aktuelle Ernährungsthemen im Bereich der Neonatologie mit freundlicher Unterstützung

Nestlé Nutrion Institute
12:15 - 13:45 Uhr RSV-Infektionen News

mit freundlicher Unterstützung

Abbott GmbH \& Co. KG

12:15 - 13:45 Uhr

Was ist neu bei Asthma im

Kindesalter?

mit freundlicher Unterstützung

MSD Sharp \& Dohme GmbH

Vorsitz:

S. Zielen (Frankfurt a. M.)

- Virusindiziertes Asthma über- oder unterschätzt? H.-G. Bresser (Bielefeld)

- Belastungsasthma bei Kindern - ernstzunehmende Belastung oder nur Belästigung?

F. Panzer (Mannheim)

- Asthmamanagement im

Kindesalter - gibt es

Prädiktoren für den

Therapieerfolg?

S. Zielen (Frankfurt a. M.)

12:15 - 13:45 Uhr

Perspektiven aktueller Impfempfehlungen

mit freundlicher Unterstützung

Sanofi Pasteur MSD GmbH

Vorsitz:

H. Schroten (Mannheim)

- Einführung

H. Schroten (Mannheim)

- „HPV-2009 - neue Heraus-

forderungen - praktische Umsetzung"

P. Hillemanns (Hannover)

- „Rotavirus-Schluckimpfung: Wo stehen wir?"

M.A. Rose (Frankfurt a. M.)

- Pertussis: Häufig bei

Erwachsenen

C. H. Wirsing von König

(Krefeld)

- Zusammenfassung

H. Schroten (Mannheim)

12:15 - 13:45 Uhr

Schwere primäre Immundefizienz

mit freundlicher Unterstützung

Orphan Europe (Germany) GmbH Vorsitz: W. Friedrich (UIm),

B. Belohradsky (München)

12:15 - 13:45 Uhr

Vom Verdacht zur Diagnose

mit freundlicher Unterstützung

CSL Behring GmbH

12:15 - 13:45 Uhr

Wichtige Aspekte der Pneumokokkenimpfung

mit freundlicher Unterstützung GlaxoSmithKline GmbH \& Co. KG Vorsitz:

R. von Kries (München) 
- Prävention systemischer Pneumokokkeninfektionen - Erreichtes und Herausforderungen R. von Kries (München)

- Aktuelle Aspekte der Ätiologie, der Klinik und der Therapie der akuten Otitis media G. Grevers (Starnberg)

- Synflorix - ein neuer Pneumokokkenimpfstoff hat sich bewährt K. Ludwig (Hamburg)

\section{2:15 - 13:45 Uhr}

Von KiTa bis Ausbildung Neue Studien zu Probiotika mit freundlicher Unterstützung Danone $\mathrm{GmbH}$

Vorsitz:

B. Koletzko (München),

M. J. Lentze (Bonn)

- Von Anfang an: Entwicklung und Zusammenspiel von Darmflora und Immunsystem

M. Blaut (Nuthetal)

- Viele Kinder - viele Infektionen: Präventiver Einsatz von Probiotika in der Kinderbetreuung Chr. P. Braegger (Zürich, Schweiz)

- Zu viel Stress? Einfluss von Probiotika auf stressinduzierte Infektanfälligkeit bei jungen Erwachsenen J. Schrezenmeir (Kiel)

\section{4:15 - 15:45 Uhr} Interaktiver Workshop zu schwierigen Impffragen (TED) mit freundlicher Unterstützung GlaxoSmithKline GmbH \& Co. KG Im Rahmen einer interaktiven Sitzung mit TED-Unterstützung werden vorbereitete sowie auch spontan aus dem Auditorium gestellte schwierige Impffragen aus dem gesamten Bereich der Vaccinologie diskutiert. So werden unterschiedlichste Themen wie beispielsweise die Weiterimpfung von untypisch vorgeimpfter Migrantenkinder, Fragestellungen zur 2maligen Varizellenimpfung oder zu neu zugelassenen Impfstoffen angesprochen.

\section{Symposien}

Freitag, 04.09.2009

\section{4:15 - 15:45 Uhr}

Chronische Krankheit und Pubertät

Sitzung der DGKJ in Zusammenarbeit mit der AG Pädiat. Psychosomatik

Vorsitz: G. Bürk (Herne),

J. Meister (Aue)

- 13 Jahre, Türkin, Rheuma - „und wer versteht mich" K. Mönkemöller (Köln)
- Der jugendliche Patient mit Diabetes mell. Typ I und rezidivierenden Ketoazidosen und Hypoglykämien "ich bin doch nicht krank" E. Lilienthal (Bochum)

- Vater dick, Mutter dick, 15-jährige Tochter dick: Adipositas als Schicksal - „bitte nicht wiegen, ich esse ganz normal" J. Mirza (Köln)

- Jugendliche Patientin mit multiplen somatoformen Beschwerden zwischen Maximaldiagnostik und Verdacht auf Simulation - „macht mir doch endlich die Schmerzen weg" D. Kunert (Kassel), M. Weckesser (Kassel)

- 17 Jahre, Epilepsie, Computerfreak - „und ich darf gar nichts, keine Disco, kein Alkohol, kein Auto, keine Lanparty - oder doch?" M. Blankenburg (Datteln), $M$. Nitsche (Göttingen)

14:15 - 15:15 Uhr Praxisrelevante Leitlinien in der Pädiatrischen Endokrinologie und Diabetologie

in Zusammenarbeit mit der AG Pädiat. Endokrinologie, AG Pädiat. Diabetologie und AG Adipositas im Kindes- und Jugendalter

Vorsitz: B. Karges (Aachen),

J. Herwig (Frankfurt a. M.)

- Kleinwuchs: Wann liegt ein Wachstumshormonmangel vor?

G. Binder (Tübingen)

- Adipositas bei Kindern und Jugendlichen:

Evidenzbasierte Therapieleitlinien

A. Moss (Ulm)

- Evidenzbasierte Leitlinien für Diabetes Mellitus im Kindes- und Jugendalter B. Karges (Aachen)

14:15 - 15:45 Uhr Review of the Year Vorsitz: G. Hansen (Hannover), M. Mall (Heidelberg)

- Update Ernährung B. Koletzko (München)

- Update Lungenerkrankungen M. Mall (Heidelberg)

- Update Allergie G. Hansen (Hannover)

- Update Infektiologie

T. Tenenbaum (Mannheim)

14:15 - 15:15 Uhr Risiken - Krisen - Identitäten Vorsitz: B. Stier (Butzbach), W.-R. Horn (Gernsbach)
- Jugend im Vollrausch? - eine klinisch-psychologische Betrachtung E. Fandler (Graz, Österreich)

- Sexualität und Gewalt bei Jugendlichen N. Weissenrieder (München)

- Jungen in der Krise - Krise der Gesellschaft? B. Stier (Butzbach)

16:15 - 18:00 Uhr

Praktizierte Jugendmedizin in Schwerpunkteinrichtungen Wie kann der Kinderarzt davon profitieren

Sitzung der DGKJ in Zusammenarbeit mit dem Forum für Jugendmedizin e.V.

Vorsitz: S. Eber (München),

E. Friederichs (Bamberg)

- Schulung von chronisch kranken Jugendlichen Zugang zu einem verbesserten (Krankheits-) Verständnis? U. Kuhnle-Krahl (Gauting)

- Schwerpunkteinrichtungen für die Pädiatrie: Zukunft für ein qualifiziertes Angebot für Jugendliche - Erfahrungen aus zwei Schwerpunktpraxen S. Eber (München), E. Friedrichs (Bamberg)

- Aufbau einer erfolgreichen interdisziplinären Jugendsprechstunde: Was können Pädiater und Allgemeinmediziner voneinander lernen? B. Hemming (Duisburg)

- Jugendliche in der Entwicklung: Wie kann ein adäquates Setting einer Praxis aussehen?

\section{R. Marx-Mollière (Mainz)}

16:15 - 17:45 Uhr

Rauchen und Gesundheit bei Kindern und Jugendlichen Vorsitz:

M. Pötschke-Langer (Heidelberg),

S. Zielen (Frankfurt a. M.)

- Welche Kinder und

Jugendliche rauchen in

Deutschland?

T. Langer (Berlin)

- Auswirkungen des Aktivund Passivrauchens a uf die Gesundheit von Kindern und Jugendlichen

M. Rosewich

(Frankfurt a. M.)

- Was wirkt in der Tabakprävention?

M. Pötschke-Langer (Heidelberg)

- Umgang mit Jugendlichen, die rauchen (insbesondere, wenn sie noch an Asthma und anderen Störungen leiden)

W.-R. Horn (Gernsbach)
16:15 - 17:45 Uhr

Primäre Prävention von Adipositas

Vorsitz:

K. E. Bergmann (Berlin),

B. Koletzko (München)

- Vorkommen, Trend und Bedeutung von Adipositas K. E. Bergmann (Berlin)

- Frühe Programmierung späterer Adipositas R. L. Bergmann (Berlin)

- Studien und Ergebnisse zur frühen Adipositasprävention B. Koletzko (München)

- Ätiologische Faktoren für die Adipositasentstehung im Schulalter

K. Zwiauer

(St. Pölten, Österreich)

- Studien und Ergebnisse zur Adipositasprävention im Schulalter

M. J. Müller (Kiel)

16:15 - 17:55 Uhr

Management und Ökonomie

für Kliniken für Kinder und Jugendliche

Vorsitz:

W. Kölfen (Mönchengladbach)

- Zukünftige Wirkungsfelder der stationären Kinder- und Jugendmedizin

B. Lettgen (Darmstadt)

- Ökonomische Chancen und Grenzen für Kinderkliniken in der DRG-Welt - Reicht das Geld für die stationäre Pädiatrie in der Zukunft? H. Schilling (Siegburg)

- Das ambulante Kinderkrankenhaus - Modell der Zukunft? Rahmenbedingungen und Einsatzmöglichkeiten für neue Strukturen F. Riedel (Hamburg)

- Ist ein Ende der Unterfinanzierung von ambulanten Leistungen in Kinderkliniken in Sicht? - Aktueller Stand und was ist zu tun? J. Scheel (Siegen)

- Hart aber fair - Wenn die Referenten auf die Wirklichkeit treffen? W. Kölfen (Mönchengladbach)

16:15 - 17:45 Uhr

Schlaf, Bewegungsmangel, Medienkonsum und Adipositas

in Zusammenarbeit mit der Dt. Gesell. für Schlafforschung und Schlafmedizin und der AG Adipositas im Kindes- und Jugendalter Vorsitz: A. Wiater (Köln) 
- Bewegungsmangel und Medienkonsum als Ursache der Adipositas - Möglichkeiten für therapeutische Ansätze?

S. Ehehalt (Tübingen)

- Einfluss von Übergewicht auf Schlaf und Atmung Pathophysiologische und diagnostische Betrachtungen

P. Brockmann (Tübingen)

- Schlafapnoen, Adipositas und Hypertonie

A. Wiater (Köln)

- Adipositas und Schlafapnoen - therapeutische Strategien

M. Urschitz (Tübingen)

- Diagnostik und Therapie der Hypoventilationssyndrome A. Kramer (Berlin)

- Einfluss von Sport auf Adipositas und Schlafqualität

M. Dworak

(West Roxbury, USA)

16:15 - 18:00 Uhr

- Historisches Symposium

Vorsitz: G. Gaedicke (Berlin),

T. Beddies (Berlin)

- Über die "standhafte Ge-

sundheit" der Kinder. Franz Anton Mai (1742-1814) und die medizinische Aufklärung in Mannheim

E. Seidler (Freiburg)

- Der Weg Friedrich Schillers

nach Mannheim

V. Hesse (Berlin)

- Jüdische Ärzte aus Deutschland als Modernisierer der akademischen Medizin in der Türkei am Beispiel Albert von Eckstein und Erich Frank

G. Gaedicke (Berlin)

- "Muttermilch ist unersetzlich" - Säuglingsfürsorge und ärztliche Stillempfehlungen im 20. Jahrhundert J. Vögele (Düsseldorf)

- "Heilgymnastik und Schulturnen" - Gesundheitsfördernde und therapeutische Bewegungskonzepte für Kinder und Jugendliche 1870 - 1930

E. Brinkschulte (Magdeburg)

\section{9:00 Uhr}

Benefizkonzert des Orchesters der Deutschen Kinderärzte
Plenarsitzung

Samstag, 05.09.2009

08:30 - 10:00 Uhr

Neurometabolik

Vorsitz:

HJ. Böhles (Frankfurt a. M.),

G. F. Hoffmann (Heidelberg)

- Einführung und Übersicht neurometabolischer Krankheitsbilder. Interaktive Show M. Duran, F. Wijburg (Amsterdam, Niederlande)

\section{Symposien}

\section{Samstag, 05.09.2009}

10:30 - 12:00 Uhr

Neurometabolische Diagnostik (Vertiefung I)

Vorsitz: A. Kohlschütter

(Hamburg), M. Kieslich

(Frankfurt a. M.)

- Klinischer Zugang neurometabolischer Erkrankungen

A. Kohlschütter (Hamburg)

- Laborchemischer Zugang neurometabolischer Erkrankungen G. F. Hoffmann (Heidelberg)

- Liquordiagnostik neurometabolischer Erkrankungen S. Vlaho (Frankfurt a. M.)

- Neurophysiologische und bildgebende Diagnostik neurometabolischer Erkrankungen

M. Kieslich (Frankfurt a. M.)

10:30 - 12:00 Uhr

Update Bewegungsstörungen

in Zusammenarbeit mit der Gesellschaft für Neuropädiatrie e.V.

Vorsitz: F. Heinen (München),

H.-J. Christen (Hannover)

- Gangstörungen im Kindesalter - Differentialdiagnostische Tipps anhand von Videos

H.-J. Christen (Hannover)

- Klassifikation, Verlauf und Intervention bei $\mathrm{CP}$

F. Heinen (München)

- Prävention von Hüftluxationen bei Kindern mit Cerebralparese V. Mall (Freiburg)

- Physiotherapeutische Verfahren unter "evidence based " Kriterien K. Müller (Meerbusch)
10:30 - 12:00 Uhr

Kontroverse Themen in der Pädiatrischen Infektiologie

in Zusammenarbeit mit der DGPI e.V. Vorsitz: R. Berner (Freiburg),

H.-I. Huppertz (Bremen)

- RSV und Asthma - wie ist die Datenlage?

J. Forster (Freiburg)

- Antibiotikaverschreibung bei Atemwegsinfektionen in der Praxis - Neue Konzepte! A. Altiner (Düsseldorf)

- Tonsillopharyngitis - Neue Leitlinie: Halsschmerzen! R. Berner (Freiburg)

10:30 - 12:00 Uhr

Die Neuronalen Ceroid Lipo-

fuszinosen

Vorsitz:

R. Steinfeld (Göttingen)

- Die NCL-Stiftung

F. Stehr (Hamburg)

- Klinische Merkmale der Varianten der spätinfantilen NCL-Erkrankung K. Reinhardt (Göttingen)

- Ökonomische Diagnostik der neuronalen CeroidLipofuszinosen A. Schulz (Hamburg)

- Breaking Bad News: Die gemeinsame Besprechung der Diagnose stellt entscheidende Weichen für die Zusammenarbeit zwischen Familie und Arzt A. Richterich (Hamburg)

- Die ärztliche Begleitung von Kindern und Jugendlichen mit JNCL

R. Niezen

(Doorn, Niederlande)

\section{0:30 - 12:00 Uhr}

Neue und alte Aspekte der

Surfactant-Therapie

Vorsitz: G. Jorch (Magdeburg),

T. Schaible (Mannheim)

- Genetische Faktoren von neonatalen Atemstörungen L. Gortner (Homburg/Saar)

- Update der Surfactantanwendung beim Frühgeborenen - Brauchen wir seltener Surfactant?

Chr. Speer (Würzburg)

- Update der Surfactantanwendung beim ARDS

L. Bindl

(Luxemburg, Luxemburg)

- Neue Surfactantpräparationen

I. Reiss (Rotterdam, NL)
Industriesymposien

Samstag, 05.09.2009

12:15 - 13:45 Uhr

Morbus Niemann-Pick Typ C

im Fokus: Standortbestim-

mung und neue therapeu-

tische Perspektiven

mit freundlicher Unterstützung

Actelion Pharmaceutical

Deutschland $\mathrm{GmbH}$

Vorsitz:

Chr. Korenke (Oldenburg),

O. Bodamer

(Salzburg, Österreich)

- Einführung Chr. Korenke (Oldenburg)

- Pathogenese und Diagnostik des Morbus Niemann-Pick Typ C

O. Bodamer (Salzburg, Österreich)

- Therapie des Morbus Niemann-Pick Typ C: wo stehen wir 2009? (klinische Studien mit Miglustat)

T. Marquardt (Münster)

- Besonderheiten in der Diagnostik und Therapie der Patienten mit Morbus Niemann-Pick Typ C: Fallbeispiele

E. Mengel (Mainz)

12:15 - 13:45 Uhr

Gerinnung - kein Buch mit 7 Siegeln

mit freundlicher Unterstützung

CSL Behring GmbH

12:15 - 13:45 Uhr

Diagnostische Strategien bei Thrombopenie und bei Verdacht auf Immundefekt mit freundlicher Unterstützung Baxter Deutschland GmbH Vorsitz:

U. Baumann (Hannover)

12:15 - 13:45 Uhr

ADHS-Therapie: Besonderheiten bei Entwicklung und Komorbiditäten

mit freundlicher Unterstützung

Shire Deutschland GmbH

Vorsitz:

K. Skrodzki (Forchheim)

- Einfluss der pharmako-

logischen Therapie auf das

Wachstum des Kindes

M. Huss (Mainz),

K. Skrodzki (Forchheim)

- Epilepsi und ADHS

K. Stollhoff (Hamburg) 
12:15 - 13:45 Uhr

Die Pneumokokkenimpfung:

Wissen was ist, wissen was

kommt

mit freundlicher Unterstützung

Wyeth Pharma GmbH

Vorsitz:

U. Heininger (Basel, Schweiz),

T. Schwarz (Würzburg)

- Erfahrungen und Erfolge der Pneumokokkenimpfung

$U$. Heininger

(Basel, Schweiz)

- Zukunft der Pneumokokkenimpfstoffe

R. R. Reinert

(Paris La Défense, F)

- Ausblick: Die Pneumo-

kokkenimpfung bei älteren

Kindern und Erwachsenen

T. Schwarz (Würzburg)

\section{2:15 - 13:45 Uhr}

Die Ernährungssituation von

Säuglingen und Kleinkindern

in Deutschland - Status quo,

Risiken, Lösungsansätze

mit freundlicher Unterstützung

Hipp GmbH \& Co. Vertrieb KG

Vorsitz: B. Koletzko (München)

- Eröffnung und Begrüßung

B. Koletzko (München)

- Nährstoffversorgung von Säuglingen und Kleinkindern: - zwischen zu viel und zu wenig

H. Heseker (Paderborn)

- Macht zu viel Eiweiß im

Kindesalter unsere Kinder dick?

Chr. Denzer (UIm)

- Ansatzpunkte zur Verbesserung der Ernährung bei Säuglingen und Kleinkindern

M.J. Lentze

- Zusammenfassung und

Verabschiedung

B. Koletzko (München)

14:15 - 15:45 Uhr Impfsymposium: HPV, Rotavirus, MMRV, MenACWY-TT

mit freundlicher Unterstützung GlaxoSmithKline GmbH \& Co. KG

Vorsitz: S. Bigl (Chemnitz)

- Vergleichende Immunologie zweier HPV-Impfstoffe T. Schwarz (Würzburg)

- Allgemeine Rotavirusimpfung - Erste Erfahrungen anderer Länder

S. Bigl (Chemnitz)

- 3 Jahre MMRV-Impfung wo stehen wir? V. Schuster (Leipzig)

- Die Entwicklung eines neuen MenACWY-TT Impfstoffs M. Knuf (Mainz)
Symposien

Samstag, 05.09.2009

- Gedeihstörung

D. Haas (Heidelberg)

14:15 - 15:45 Uhr

Neurometabolische Krankheitsbilder (Vertiefung II)

Vorsitz:

P. Freisinger (München),

R. Steinfeld (Göttingen)

- Leukodystrophien

H. Rosewich (Göttingen)

- Neuronale Zeroidlipofuszi-

nose

R. Steinfeld (Göttingen)

- Lysosomale Speicherkrankheiten

E. Mengel (Mainz)

- Mitochondriopathien

P. Freisinger (München)

14:15 - 15:45 Uhr

Langzeitfolgen nach Behand-

lung von Krebskrankheiten im

Kindes- und Jugendalter

in Zusammenarbeit mit der Gesell. f. Pädiat.

Onkologie und Hämatologie gem. e.V. und der AG zur Betrachtung der Langzeit- und

Spätfolgen von Krebsbehandlungen im Kindesalter

Vorsitz: U. Creutzig (Münster)

G. Calaminus (Münster)

- Epidemiologie von Zweit-

malignomen nach Krebs im Kindesalter

P. Kaatsch (Mainz)

- Einführung: Die Struktur der Langzeitnachsorge und

Einführung zur Kardiotoxizität

U. Creutzig (Münster)

- Kardiotoxizität und präventive Ansätze

T. Langer (Erlangen)

- Kardiale Spätfolgen nach mediastinaler Radiotherapie

- Beispiel Morbus Hodgkin

im Kinder- und Jugendalter G. Schellong (Münster)

- Pulmonale Spätfolgen nach Radiotherapie: Korrelation mit Dosis und bestrahltem

Volumen

T. Bölling (Münster)

- Überlebensqualität - Reflexion, langfristige Lebensperspektive

G. Calaminus (Münster)

14:15 - 15:55 Uhr

Leitsymptome angeborener Stoffwechselerkrankungen

Vorsitz:

H.-G. Koch (Braunschweig),

T. Marquardt (Münster)

- Ketonurie

J.-O. Sass (Freiburg)

- Lebererkrankungen

J. Häberle (Zürich, Schweiz)

- Bewusstseinsstörung

Chr. Korenke (Oldenburg)
14:15 - 16:00 Uhr

Klinisches Spektrum der

Patienten mit Ataxia telangiectasia

Vorsitz:

S. Zielen (Frankfurt a. M.),

R. Schubert (Frankfurt a. M.)

- Klinik, Diagnostik und Versorgung der Patienten S. Zielen (Frankfurt a. M.)

- Mutations- und Expressionsanalysen des ATM-Gens T. Dörk-Bousset (Hannover)

- Neurodegeneration bei AT M. Kieslich (Frankfurt a. M.)

- Einfluss von Wachstumshormonen auf die Dystrophie und Neurodegeneration im Atm Knock-out Mausmodell R. Schubert (Frankfurt a. M.)

14:15 - 15:45 Uhr

Review of the Year

Vorsitz: J. Gärtner (Göttingen),

Chr. Speer (Würzburg)

- Update Neonatologie Chr. Speer (Würzburg)

- Update Neurologie F. Heinen (München)

- Update Neurometabolik J. Gärtner (Göttingen)

- Fetale Programmierung J. Dötsch (Erlangen)

16:15 - 17:45 Uhr

Neonatologie und Neurologie Vorsitz: R. F. Maier (Marburg),

E. Mildenberger (Mainz)

- Neurologische Überwachung auf der NICU C. Roll (Datteln)

- State of the Art: Behandlung der Asphyxie E. Mildenberger (Mainz)

- Der neurometabolische Notfall des Neugeborenen

S. Vlaho (Frankfurt a. M.)

16:15 - 18:00 Uhr

Allogene Stammzelltransplantation und Zelltherapie Vorsitz:

T. Klingebiel (Frankfurt a. M.), St. Burdach (München)

- Die haploidentische Stammzelltransplantation - Plattform für adoptive Zelltherapie

P. Bader (Frankfurt a. M.)

- T-Zelltherapien zur Rezidivprävention nach allogener Stammzelltransplantation C. Rössig (Münster)
- T-Zelltransfer zur Behandlung von CMV, ADV und EBV-Virusinfektionen nach allogener Stammzelltransplantation

T. Feuchtinger (Tübingen)

- Transplantation von mesenchymalen Stromazellen - von der Biologie zum

klinischen Einsatz

R. Meisel (Düsseldorf)

16:15 - 18:00 Uhr

Radiologische Diagnostik bei kongenitalen Fehlbildungen und Stoffwechselstörungen

in Zusammenarbeit mit der Gesell. für

Pädiat. Radiologie

Vorsitz: T. Diehm (Mannheim),

S. Ley (Heidelberg)

- Fetal-MRT, Möglichkeiten und Relevanz

D. Klee (Düsseldorf)

- Spinale Fehlbildungen

- Diagnostik in Sonografie und MRT

J.-P. Schenk (Heidelberg)

- Bildgebung bei Darmatresien und kongenitalen enteralen Obstruktionen J.-P. Schenk (Heidelberg)

- Zerebrale MR-Spektroskopie - ein zusätzlicher Gewinn?

I. Harting (Heidelberg)

16:15 - 17:45 Uhr

Frühentlassung des Neugeborenen aus der Geburtsklinik - praktische Konsequenzen für den Niedergelassenen

Kinderarzt

Vorsitz: A. Pizzuli (Köln),

R. Schlößer (Frankfurt a. M.)

- Zahlen und Fakten zu einer veränderten Politik B. Misse/witz (Eschborn)

- Erkrankungen, die wir lieber in der Klinik entdecken würden

J. Möller (Saarbrücken)

- Das erweiterte Neugeborenenscreening: Erfolge und neue Herausforderungen G.F. Hoffmann (Heidelberg)

- Vorsorgepflicht und Verantwortung im medizinischen "work sharing" 
16:15 - 17:15 Uhr

Neurologische und neurometabolische Erkrankungen des Neugeborenen

Vorsitz: S. Stockler-Ipsiroglu (Vancouver, Kanada),

M. Kieslich (Frankfurt a. M.)

- Differentialdiagnose und genetische Diagnostik des "floppy infant"

R. König (Frankfurt a. M.)

- Angeborene Hirnfehlbildungen

U. Stephani (Kiel)

- Neonatale Krampfanfälle S. Stockler-Ipsiroglu

(Vancouver, Kanada)

16:15 - 17:45 Uhr Immundefekt und Lymphoproliferation

Vorsitz: S. Ehl (Freiburg),

$N$. Wagner (Aachen)

- Fieber und Lymphoproliferation: Typische Fälle,

Diagnostik und Therapie

- Fieber und Lymphoproliferation: Immunologie und Genetik

S. Ehl (Freiburg)

- Autoimmunität und Lymphoproliferation M. Hönig (UIm)

- Infektanfälligkeit und Lymphoproliferation K. Warnatz (Freiburg)

16:15 - 17:45 Uhr Aktueller Wissensstand zum Tourette Syndrom - von der Diagnostik bis zur Therapie Vorsitz:

V. Roessner (Göttingen),

A. Münchau (Hamburg)

- Diagnostik und Epidemiologie des Tourette Syndroms K. Müller-Vahl (Hannover)

- Pathophysiologie des Tourette Syndroms

A. Münchau (Hamburg)

- Therapie des Tourette Syndroms

I. Neuner (Aachen)

- Besonderheiten des Tourette Syndroms in Kombination mit ADHS und Zwängen - von der Diagnostik zur Therapie

V. Roessner (Göttingen)

17:30 - 19:00 Uhr

Mitgliederversammlung der

Deutschen Gesellschaft für

Kinder- und Jugendmedizin

19:30 Uhr

Gesellschaftsabend im Lan-

desmuseum für Technik und

Arbeit
Plenarsitzung

Sonntag, 06.09.09

\section{9:00 - 10:25 Uhr}

Migrantenmedizin

gemeinsame Sitzung mit der Deutschen

Gesellschaft für Sozialpädiatrie und Jugend-

medizin e.V.

Vorsitz: M. Knipper (Gießen),

F. Aksu (Datteln)

- Transkulturalität aus der Sicht der Pädiatrie HJ. Böhles (Frankfurt a. M.)

- Soziale Auswirkungen der

Transkulturalität aus der

Sicht der Pädiatrie

H. M. Straßburg (Würzburg)

- Transkulturalität in der Zeit der Globalisierung

O. S. Ipsiroglu

(Vancouver, Kanada)

- Interdisziplinäre Aspekte der medizinischen Versorgung der Migranten M. Knipper (Gießen)

- Erfahrungen aus der täg-

lichen Praxis

F. Aksu (Datteln)

\section{Symposien}

\section{Sonntag, 06.09.09}

11:00 - 13:00 Uhr

Zweispracherwerb und vorsprachliche Entwicklung

Vorsitz:

K. Wermke (Würzburg)

- Infants get the rhythm - sprachrelevante Leistungen

Neugeborener und junger Säuglinge

K. Wermke (Würzburg)

- Prägen intrauterine Hörerfahrungen die Lautproduktion? Ein Vergleich französischer und deutscher Neugeborener

B. Mampe (Würzburg)

- Bilinguale Kindergärten: Was bringt's? Erfahrungen und Forschungsergebnisse zum frühen Fremdsprachenerwerb

A. Steinlen (Kiel)

- Kein Bilingualer gleicht dem anderen - der Einfluss der kognitiven Kontrolle auf den Gebrauch von Sprachen J. Festman (Magdeburg)
11:00 - 12:30 Uhr

Pädiatrische Tropen- und

Reisemedizin

Vorsitz:

M. Krawinkel (Gießen)

- „Neue Influenza /

Schweinegrippe"

W. Haas (Berlin)

- BlueXQ gegen Malaria

O. Müller (Heidelberg)

- Family Care instead of Child Care for HIV-infected families

M. E. Swai

(Moshi, Tanzania, Afrika)

- Behandlung des schwer mangelernährten Kindes - ambulant oder stationär M. Krawinkel (Gießen)

11:00 - 12:30 Uhr

Traumatisierung und Traumafolgen bei Flüchtlingskindern B. Möller (Hamburg),

S. Schlüter-Müller

(Frankfurt a. M.)

11:00 - 12:30 Uhr

Kinder- und Jugendgynäkologie bei Migranten - einschließlich Genitalverstümmelung

11:00 - 12:15 Uhr

Praxisrelevante Leitlinien in der Pädiatrischen Endokrinologie und Diabetologie

in Zusammenarbeit mit der AG Pädiat. Endokrinologie, AG Pädiat.Diabetologie und AG Adipositas im Kindes- und Jugendalter

Vorsitz: H. Krude (Berlin),

S. Heger (Hannover)

- Hodenhochstand: Welches Vorgehen ist richtig? N. Albers (Osnabrück)

- Konfirmationsdiagnostik bei positivem Screening-Befund für 21-Hydroxylasemangel (adrenogenitales Syndrom) K. Mohnike (Magdeburg)

- Konnatale Hypothyreose: Evidenzbasiertes therapeutisches Vorgehen H. Krude (Berlin)

- Störungen der Geschlechtsentwicklung: Welches diagnostische und therapeutische Vorgehen ist richtig? P. M. Holterhus (Kiel)
11:00 - 13:00 Uhr

Aktuelle Impffragen:

Die STIKO informiert

in Zusammenarbeit mit dem Robert Koch-

Institut und der Ständigen Impfkommission

Vorsitz: Y. Deleré (Berlin),

F. Zepp (Mainz)

- Wie entstehen

Impfempfehlungen?

D. Matysiak-Klose (Berlin)

- Kritische Abwägungen zu der Impfstrategie gegen

Varicella-Zoster-Virus

T. Mertens (Ulm)

- Änderungen der PertussisImpfempfehlung: Der schwierige Weg zu fallenden Inzidenzen $R$. von Kries (München)

- Krankheitslast von RotaViren in Deutschland M. Wiese-Posselt (Berlin)

\section{1:00 - 13:00 Uhr}

Ciliopathien in der Kindernephrologie Vorsitz:

D. E. Müller-Wiefel (Hamburg)

- Einleitung

H. Omran (Freiburg)

- Nephronophthise

C. Höfele (München)

- Rezessive Cystennieren

D. Haffner (Rostock)

- Dominante Cystennieren

Chr. von Schnakenburg (Freiburg)

- Das Bardet-Biedl-Syndrom als Modellerkrankung syndromaler Ziliopathien C. Bergmann (Aachen)

\section{Vorläufiges Programm der DGKCH}

\section{Workshops \\ Donnerstag, 03.09.2009}

14:00 - 16:00 Uhr

Trauma Crash-Kurs:

Fixateur externa

Vorsitz: W. L. Linhart

(Graz, Österreich)

16:00 - 18:00 Uhr Nachsorge operierter Ösophagusatresien

Vorsitz:

J. Trompelt (Niederhausen),

St. Kellnar (München) 


\section{Symposien}

Freitag, 04.09.2009

09:00 - 11:00 Uhr

Schädel-, Hals- und Thorax-

chirurgie

Vorsitz: F. Schier (Mainz),

A. Rokitansky (Wien, A)

- Komplikationsmanagement

bei Erkrankungen des

Ösophagus

F. Schier (Mainz)

- Komplikationsmanagement bei Zwerchfellhernienoperationen

S. Hosie (München)

- Komplikationen und ihre

Therapie bei Trichterbrustoperationen

A. Rokitansky (Wien, A)

- Manometrische Evaluation

nach Foker - Rekonstruktion

bei langstreckiger Öspha-

gusatresie

R. Wachowiak (Leipzig),

U. Bühligen (Leipzig),

U. Rolle (Frankfurt a. M.)

J. Foker (Minneapolis, USA)

H. Till (Leipzig)

- Kasuistik zum Verfahrens-

wechsel nach wiederholter

Nuss-Korrektur

S. Weih, R. Romero,

S. Holland-Cunz

(Heidelberg)

B. Reingruber (Regensburg)

\section{1:30 - 12:00 Uhr}

Endoskopie und MIC

Vorsitz: J. Fuchs (Tübingen),

H. Till (Leipzig)

- Komplikationsmanagement bei minimalinvasiven Eingriffen des Thorax-Raumes und Abdomens

J. Fuchs (Tübingen)

- Arbeitspausen bei komplexer, minimalinvasiver Kinderchirurgie: Deutlicher Vorteil für den Chirurgen ohne Nachteil für das Kind B. M. Ure, M. Schneider, C. Engelmann, J. Dingemann (Hannover)

\section{4:15 - 16:00 Uhr}

Abdominalchirurgie Teil I Vorsitz: M. Höllwarth (Graz),

C. Petersen (Hannover),

A. Pintér (Pecs, Ungarn)

- Komplikationsmanagement nach hepatobiliären Operationen

C. Petersen (Hannover)

- Chirurgische Therapie von Komplikationen bei M. Crohn und Colitis Ulzerosa K.-L. Waag (Mannheim)
- Die Behandlung von Komplikationen nach Chirurgischer Korrektur Anorektaler Fehlbildungen A.M. Holschneider (Bergisch-Gladbach)

- Necrotizing Enterocolitis and the Surgeon

A. Bianchi (Manchester, GB)

16:30 - 18:15 Uhr

Abdominalchirurgie Teil II

Vorsitz: M. Höllwarth (Graz),

A. Pintér (Pécs, Ungarn)

- A structured Plan for the Short Bowel child

A. Bianchi

(Manchester, GB)

- Indikationen, operative Techniken und Resultate der Darm-Transplantation im Kindesalter

R. Gruessner

(Tucson, AZ, USA)

- Behandlung von Komplikationen nach $\mathrm{M}$. Hirschsprung-Operationen S. Holland-Cunz (Heidelberg)

- Nabelschnurplastik bei großen angeborenen Bauchwanddefekten R. Werbeck, Hamburg, J.L. Koltai (Frankfurt a.M.)

- Freie Vorträge

Der Kinderchirurgische

Stammtisch findet ab 19:30

Uhr statt

\section{Symposien}

Samstag, 05. 09.2009

09:00 - 10:10 Uhr

Kinderonkologie

Vorsitz:

D. von Schweinitz (München), W. Barthlen (Greifswald)

- Chirurgische Strategien bei Viszeraltumoren im Kindesalter

D. von Schweinitz (München)

- Neuroendokrine Tumoren der Appendix bei Kindern und Jugendlichen - Ergebnisse und Empfehlungen der GPOH-MET-Studie N. Boxberger, I. Leuschner, D. von Schweinitz,

A. Redlich, P. Vorwerk

- Erweiterte Hemihepatektomie oder Lebertransplantation? Leberchirurgie bei ausgedehnten Hepatoblas tomen - Zwei eigene Fälle S. Holland-Cunz, M. Chmelnik, P. Günther J. Schmidt (Heidelberg)
- Komplikationen und ihr Management eines riesigen Steißteratoms

Chr. Heinick (Jena)

- Freie Vorträge

10:30 - 12:00 Uhr

Kinderurologie

Vorsitz: Th. Boemers (Köln),

C. Leriche (UIm)

- Kinderchirurgische und kinderurologische Behandlung der Kloakenfehlbildungen

Th. Boemers (Köln)

- Complications following

Augmentation Gastro- and Enterocystoplasties

A. Pintér (Pecs, Ungarn)

- Urolithiasis im

Säuglingsalter

W. Kluwe (Bern),

B. Utsch (Berlin)

- SILS (single incision laparoscopic surgery) Decortikation einer symptomatischen Nierenzyste beim 14-jährigen Jungen

R. Wachowiak,

J.-U. Stolzenburg,

R. Metzger, Ü. Bühligen,

H. Till (Leipzig)

- Rekto-labiale Fistel eines Säuglings - unerwartete Ursache eines „BartholinAbszesses"

G. Cernaianu,

M. Hemminghaus,

M. Lutz, F. Niemann,

R.-B. Tröbs (Herne)

- Freie Vorträge

12:00 - 13:00 Uhr

Sitzung der Kinderchirurgischen Assistenten

12:00 - 14:00 Uhr

MGV des Berufsverbandes der niedergelassenen Kinderchirurgen

14:15 - 16:00 Uhr

MGV der Deutschen Gesellschaft für Kinderchirurgie

16:00 - 17:30 Uhr MGV AG Kinderurologie

Symposien

Sonntag, 06. 09.2009

09:30 - 10:30 Uhr

Postervorträge

Vorsitz:

U. Rolle (Frankfurt a. M.),

M. Jainsch (Frankfurt a. M.)
11:00 - 12:30 Uhr

Kindertraumatologie

Vorsitz: L. von Laer (Berlin),

P. Illing (Kassel)

- Komplikationen und deren Management L. von Laer (Berlin)

- Komplikationsmanagement bei Ellenbogenfrakturen L. Wessel (Mannheim)

- 4jähriger im Kofferband eingeklemmt - Schwere Degloving-Verletzung der Füße

R. Künzel (Köln)

- Gasbrandinfektion im Kindesalter - provokant oder schockierend? $K$. Rothe (Düsseldorf)

- Freie Vorträge

\section{Vorläufiges \\ Programm der DGSPJ}

Workshops

Donnerstag, 03.09.2009

14:30 - 17:30 Uhr

Die Bayley-Scales of Infant

Development

Vorsitz: P. Zeitler (Würzburg)

14:30 - 17:30 Uhr

Möglichkeiten der unterstützten Kommunikation bei „nicht sprechenden" Kindern und Jugendlichen

Vorsitz:

A. Rothmayr (Frankfurt a. M.), L. Mutio (Duisburg)

14:30 - 17:30 Uhr

Hilfsmittelversorgung

Vorsitz: A. Oberle (Stuttgart)

- Hilfsmittelversorgung für Patienten mit Spina bifida T. Michael (Berlin)

- Hilfsmittelversorgung für Patienten mit Bewegungsstörungen

S. Steinebach (Bonn)

14:30 - 17:30 Uhr

Neurokutane Syndrome Vorsitz:

G. Kurlemann (Münster)

14:30 - 17:30 Uhr

Hilfsmittelversorgung von Kindern mit Sehbeeinträchtigungen

Vorsitz: M. Henrich (Neuwied) 
14:30 - 17:30 Uhr

Kinderschutz

Vorsitz:

H. Philippi (Frankfurt a. M.)

- Interdisziplinärer Kinderschutz in der Medizin B. Herrmann (Kassel)

- Prävention von Gewalt durch frühe Förderung von Bindungssicherheit K.-H. Brisch (München)

- Kinderschutz von Anfang an aus der Sicht einer Frauenklinik

B. Filsinger (Ludwigshafen)

- Aufbau eines flächendeckenden Netzes früher Hilfen im Gesundheitswesen. Erste Ergebnisse über ein Multicenterprojekt Kinder ZUKUNFT NRW W. Kratzsch (Düsseldorf)

14:30 - 17:30 Uhr

Kinder mit Erfahrung von Migration in der Sozialpädiatrie - Aspekte für den Öffentlichen Gesundheitsdienst, Sozialpädiatrische Zentren und Rehakliniken

Vorsitz:

J. Oepen (Bad Kreuznach),

U. Horacek (Recklinghausen),

H. Hollmann (Bonn)

14:30 - 17:30 Uhr

Sprachentwicklungsverzögerung: Früherkennung und Prognose von Late Talkers

- Früherkennung von Sprachentwicklungsstörungen bei der U7 mit dem SBE-2KT: Ergebnisse einer Nachuntersuchung mit drei Jahren

K. Ullrich,

W. von Suchodoletz (München)

- Diagnostische Zuverlässigkeit eines Elternfragebogen für die U7a bei der Erfassung von Kindern mit Sprachentwicklungsstörungen S. Tippelt,

W. von Suchodoletz

(München)

- Die Entwicklung von Late Talkers ohne bzw. mit Sprachverständnisstörungen S. Sachse (U/m)

W. von Suchodoletz

(München)

- Prädikatoren für die Sprachentwicklung von Late Talkers bis zum Einschulungsalter

P. Kühn,

W. von Suchodoletz

(München)
- Beziehungen zwischen Defiziten im auditiven sensorischen Gedächtnis und der Sprachentwicklung von Late Talkers N. Großheinrich, S. Kademann, W. von Suchodoletz (München)

\section{Symposien}

Freitag, 04.09.2009

\section{0:30 - 12:00 Uhr}

Hilfsmittelversorgung

Vorsitz: H. Hollmann (Bonn),

T. Michael (Berlin)

- Allgemeine Prinzipien der Hilfsmittelversorgung

M. Schmidt Ohlemann (Bad Kreuznach)

- Verordnungssystematik der Hilfsmittelversorgung anhand exemplarischer Fallvorstellungen B. Stein-Bayet (Landstuhl)

S. Steinebach (Bonn)

K. Happes (Heidelberg

12:15 - 13:30 Uhr

"neues musik forum" Musikalische Hochbegabung im Kindesalter

Vorsitz:

U. Stiff (Kleinmachnow), H. Hollmann (Bonn)

- Musikalischer Beitrag: live $H$. Berg (Berlin, Violine (Orchester-Akademie der Berliner Philharmoniker))

- Grußworte

W. Kiess (Leipzig)

- Musizieren als Plastizitätsmotor - Zur Hirnphysiologie des kindlichen Musizierens E. Altenmüller (Hannover)

- Neurobiologische Reifungsprozesse bei musikalischer (Hoch)Begabung:

Verschwinden assoziierte Mitbewegungen (AM) beim Instrumentalspiel A. Seidler (Lörrach)

- Begabung und Hochbegabung in der Musik H. Gembris (Paderborn)

- Entwicklung musikalischer Hochleistung

A. Lehmann (Würzburg)

- Musikalischer Beitrag: live H. Berg (Berlin, Violine (Orchester-Akademie der Berliner Philharmoniker)) Konzertprogramm:

J.S. Bach: Partita für Violine solo Nr. 2 d-Moll BWV 1004, E. Ysä̈e: Sonate für Violine solo e-Moll Op. $27 \mathrm{Nr} .4$
14:15 - 15:45 Uhr

Selbsthilfegruppen in der Sozialpädiatrie

Vorsitz:

Chr. Wollstädter (Mainz)

R. Schmid (Aschaffenburg)

- Selbsthilfe: ein immer wieder neuer Anspruch an die Gesellschaft

A. Mund (Königswinter)

- Eltern-Selbsthilfe und Sozialpädiatrie: Wo müssen künftig die Weichen gestellt werden?

R. Schmid (Aschaffenburg)

- Berufsfindung bei Teilleistungsstörungen: Auf der Suche nach Hilfe und Helfer W. Fuchs-Lambrix (Ludwigshafen)

- Wie kann die ambulante und stationäre Versorgung von Patienten mit Spina bifida durch Mitwirkung von Selbsthilfegruppen verbessert werden? B. Trapp (Bodenheim)

- Wie sehen die Erwartungen von Selbsthilfegruppen an klinischen Versorgungseinrichtungen aus der Selbsthilfe Tuberöser Sklerose Deutschland aus? B. Walther (Owsschlag), Chr. Hertzberg (Berlin)

16:15 - 17:30 Uhr

Qualitätssicherung in der Sozialpädiatrie

Vorsitz: Chr. Fricke (Hamburg),

P. Borusiak (Wuppertal)

- Zertifizierung von SPZAmbulanzen

Chr. Fricke (Hamburg)

- Autismus

A. Aisch (Regensburg)

- Diagnostische Standards: Psychologische Testverfahren

F. Paulus (Homburg/Saar)

17:30 - 19:00 Uhr

Mitgliederversammlung der Deutschen Gesellschaft für Sozialpädiatrie und Jugendmedizin

\section{Symposien}

Samstag, 05.09.2009

10:30- 12:00 Uhr

ADS

gemeinsame Sitzung mit dem BV. Kinderkrankenpflege Deutschland e.V. Vorsitz:

H. M. Straßburg (Würzburg),

E. Zoller (Hannover)

- ADHD: Diagnosis, Subtyping, Impairments and Clinical Issues in Transition to Adolescence

R. Barkley (Syracuse, USA)
14:15 - 15:45 Uhr

KJGD und niedergelassene Kinderärzte: Kooperation für bessere Versorgung

Vorsitz: R. Klein (Saarbrücken)

$U$. Horacek (Recklinghausen)

- Verbesserter Kinderschutz

durch erhöhte Rate der

Teilnahme an den Kinderfrüherkennungsuntersuchungen? Gesetzliche Maßnahmen und erste Erfahrungen aus dem Saaland

L. Gortner (Homburg/Saar)

- Impfprävention im Land Brandenburg - eine erfolgreiche Kooperation G. Ellsäßer (Zossen)

- Von der Integration zur Inklusion - Was Ärzte über den Paradigmenwechsel bei der Förderung von Kindern und Jugendlichen mit Behinderung im Schulbereich wissen sollten

E. Zimmermann (Bremen)

\section{6:15 - 18:00 Uhr}

Geschichte der Sozialpädiatrie Vorsitz:

H. M. Straßburg (Würzburg), Chr. Kretzschmar (Dresden)

- Kaiserin Auguste ViktoriaHaus Berlin „Die Deutsche Vereinigung für Säuglingsund Kleinkinderschutz 1909 - 1934"

H. Wegmann (Berlin)

- „.... dass wir reife Menschen unserer Zeit sind."

(U. Köttgen) - die Vergegenwärtigung der NS"Kindereuthanasie" in der deutschen Sozialpädiatrie S. Topp (Gießen)

- Sozialpädiatrie in der DDR R. Eulitz (Reifenstein)

- Soziale Pädiatrie - einst und jetzt

H. M. Straßburg (Würzburg)

\section{Symposien \\ Sonntag, 06.09.2009}

11:00 - 12:30 Uhr

Versorgung von Jugendlichen mit geistiger Behinderung/ Freie Themen

Vorsitz: H. Bode (UIm), C. Wurst (Suhl)

- ICF-Philosophie im diagnostischen Prozess eines SPZDarstellung eines pragmatischen Ansatztes

A. Weber-Sannwald,

H. Philippi (Frankfurt a.M.) 
Symposien

Freitag, 04.09.2009

11:00 - 15:00 Uhr

Kolloquium: Pflegediagnostik

- Grundlage für die Ermittlung des Schulungs- und Beratungsbedarfs von Kindern und ihren Bezugspersonen

15:30 - 17:00 Uhr

Mitgliederversammlung des Berufsverbandes Kinderkrankenpflege Deutschland e.V. (BeKD e.V.)

\section{Symposien}

Samstag, 05.09.2009

08:30 - 09:00 Uhr

Begrüßung

09:00 - 10:30 Uhr

Ethische Dilemmata in der Neonatologie

10:30 - 12:00 Uhr

ADS

gemeinsame Sitzung mit der Deutschen Gesellschaft für Sozialpädiatrie und Jugendmedizin e $V$.

Vorsitz: H. M. Straßburg

(Würzburg)

E. Zoller (Hannover)

- ADHD: Diagnosis, Subtyping, Impairments and Clinical Issues in Transition to Adolescence

R. Barkley (Syracuse, USA)

13:30 - 15:00 Uhr Bewältigung von Krisensituationen im klinischen Alltag eine interdisziplinäre Herausforderung

Palliativcare in der akut-stationären Versorgung von Kindern und Jugendlichen

15:15 - 16:45 Uhr

Somatoforme Störungen im Kindes- und Jugendalter (Bauchschmerzen, Kopfschmerzen...)

17:00 - 18:00 Uhr

Berufspolitik

Pflegestützpunkte (SGB XI/V)

\section{ORCHESTER DER DEUTSCHEN KINDERÄRZTE} BENEFIZKONZERT

zu Gunsten der Rudolf Pichlmayr Stiftung für Kinder und Jugendliche vor und nach Organtransplantation

anlässlich der 105. Tagung der Deutschen Gesellschaft für Kinder- und Jugendmedizin in Mannheim

\section{am Freitag, den 4. September 2009 um 19.00 Uhr im Congress Center Rosengarten, Mannheim}

\section{Programm:}

Johannes Brahms:

Klavierkonzert Nr. 1, d-moll

Samuel Barber:

Dmitri Schostakowitsch: Adagio for Strings Op. 11

Solistin:

Musikalische Leitung: Symphonie Nr. 9, Op. 70, Es-Dur Katharina Treutler, Paris

Prof. Manfred Fabricius, Berlin

Eintrittspreise: 10 ,- bis $30,-€$

Kartenvorverkauf bei Anmeldung zum Kongress und Mannheimer Vorverkaufsstellen

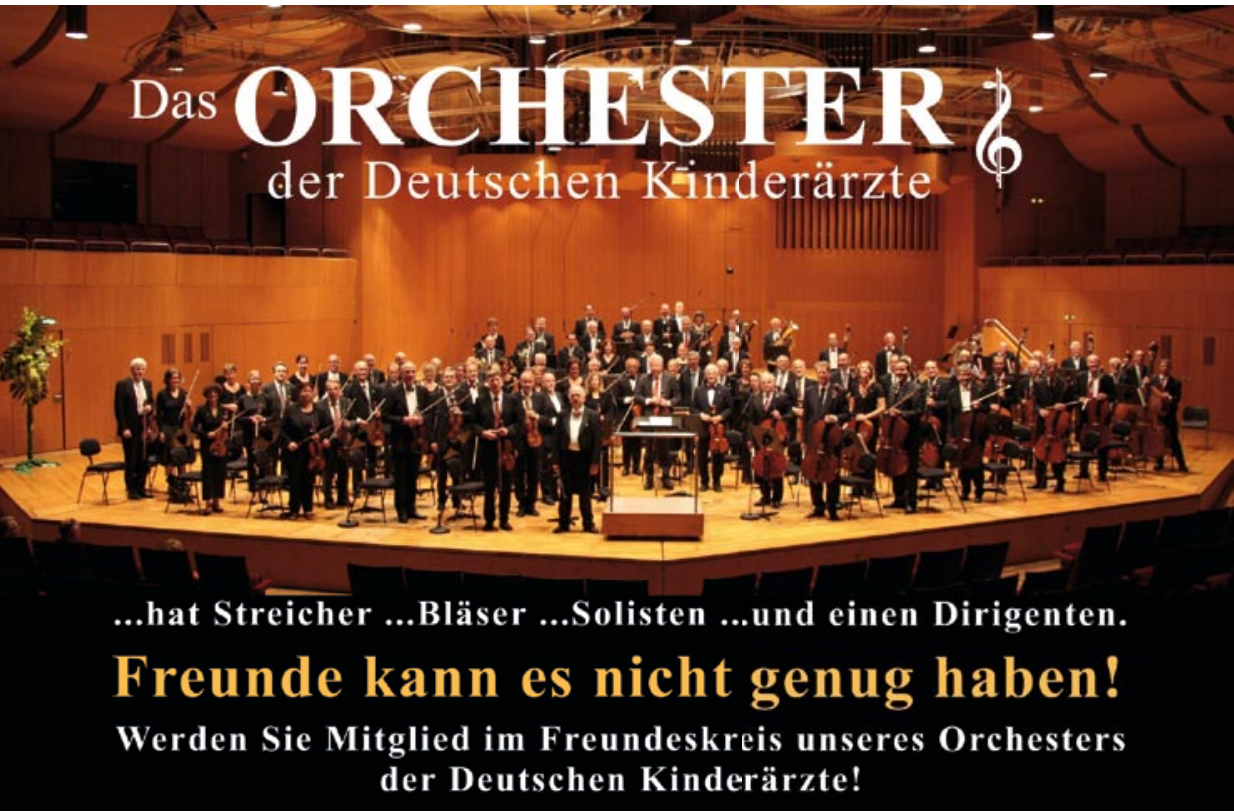

Jahresbeitrag für Mitglieder: 25,- $€$

Anmeldung bei Herrn Hans-Ulrich Laar, Joh. Seb. Bach-Str. 3, 82049 Pullach 


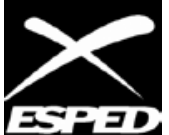

\section{Laufende Datenerhebungen im ESPED-System}

\section{Häufigkeit von plötzlichen Todesfällen und schweren lebensbedrohlichen Ereignissen ohne erkennbare Ursache am 1. Lebenstag}

Die Häufigkeit des plötzlichen Kindstodes ist in den letzten Jahren zurückgegangen. Dies hängt v. a. mit der Aufklärung der Bevölkerung über vermeidbare Risikofaktoren wie Bauchlage, Rauchen und Überwärmung zusammen. Die meisten Kinder versterben zwischen dem 2. und 4. Monat. Wenn auch seltener, so können plötzliche und unerwartete Todesfälle oder lebensbedrohliche Ereignisse auch schon am 1. Lebenstag auftreten [1-5]. $\mathrm{Zu}$ Häufigkeit und Risikofaktoren dieser Ereignisse liegen bislang jedoch kaum Daten vor.

Ziel dieser Studie ist es, solche Ereignisse ein Jahr lang deutschlandweit zu erfassen und durch Informationen zum Kind und zu den Umständen des Ereignisses evtl. Risikofaktoren hierfür zu identifizieren.

Ein plötzlicher Kindstodesfall wird hier definiert als plötzliches Versterben eines reifen Neugeborenen (Gestationsalter $\geq 37$ Schwangerschaftswochen) am 1. Lebenstag nach initial guter Adaptation (10 Minuten-APGAR mind. 8), für das sich aus Todesumständen und Obduktion keine hinreichende Erklärung (z.B. Sepsis, Herzfehler) findet.

Ein schweres lebensbedrohliches Ereignis wird definiert als akuter Zyanose- oder Blässezustand mit Bewusstlosigkeit, bei dem der hinzugerufene Arzt eine Beutelventilation, Intubation oder/ und Herzdruckmassage für notwendig hält und für das sich in der anschließenden Diagnostik keine hinreichende Erklärung findet.
Wir hoffen, im Rahmen dieser Studie vermeidbare Risikofaktoren für obige Ereignisse zu identifizieren, um in der Zukunft durch geeignete Präventionsmaßnahmen solchen Ereignissen besser vorbeugen zu können.

Bitte melden Sie jedes Ereignis an die ESPED-Zentrale. Dies betrifft auch Fälle, bei denen Sie in einer von Ihnen betreuten Geburtsklinik von einem plötzlichen Todesfall am 1. Lebenstag erfahren, der aber nicht (mehr) zur Aufnahme in eine Kinderklinik führte.

Nach der Meldung erhalten Sie einen anonymisierten Fragebogen zur Erfassung der wichtigsten klinischen und epidemiologischen Angaben, den Sie bitte ausgefüllt an uns zurücksenden; außerdem bitten wir um Zusendung des Arztbriefes und wichtiger Ergebnisse durchgeführter Diagnostik (z.B. Obduktionsbericht, mikrobiologische Untersuchungen) in anonymisierter Form (mit Schwärzung personenbezogener Angaben wie Name, Geburtsdatum, Adresse). Aufgrund der Anonymisierung aller Daten ist die Einholung des elterlichen Einverständnisses für diese Untersuchung nicht erforderlich.

\section{Studienleitung:}

Dr. med. Anette Poets, Abteilung Neonatologie,

Universitätskinderklinik Tübingen,

Calwerstr. 7, 72076 Tübingen

Tel: 07071 - 2984742,

anette.poets@

med.uni-tuebingen.de

\section{Literatur}

1. Branger B, Savagner C, Winder N. Eleven cases of early neonatal sudden death or near death of full term and healthy neonates in maternity wards. J Gynecol Obstet Biol Reprod (Paris) 2007;36:671-9
2. Espagne $S$, Hamon I, Thiebaugeorges 0 , Hascoet JM. Sudden death of neonates in the delivery room. Arch Pediatr. 2004;11(5):436-9

3. Gatti $\mathrm{H}$, Castel $\mathrm{C}$, Andrini P, Durand $P$, Carlus C, Chabernaud JL, Vial M, Dehan M, Boithias C. Cardiorespiratory arrest in full term newborn infants: six case reports. Arch Pediatr. 2004;11(5): 423-5

4. Rodriguez-Alarcón J, Malcho JC, Linares A, Aranguren G, Quintanilia M, et al. Early neonatal sudden death or near death syndromes. An epidemiological study of 29 cases. Acta Paediatr 1994;83:704-8

5. Dageville C, Pignol J, de Smet S. Very early neonatal apparent life-threatening events and sudden unexpected deaths: incidence and risk factors. Acta Paediatr 2008;97:866-9

\section{Multiple Sklerose im Kindes- und Jugendalter}

MS ist die häufigste zur Behinderung führende neurologische Erkrankung im jungen Erwachsenenalter, die das Gehirn und das Rückenmark betrifft und dort zur Bildung von sogenannten Plaques führt, in denen die Isolationsschicht der Nervenfasern zerstört wird. Erst in den 8oer Jahren wurde gezeigt, dass neben Erwachsenen auch Kinder und Jugendliche an einer MS erkranken können. Klinischer Verlauf, Prognose und Ursachen der MS im Kindes- und Jugendalter sind allerdings noch wenig untersucht. Es wird angenommen, dass die MS im Kindes- und Jugendalter vor allem eine autoimmunologische Erkrankung ist, dass aber genetische Einflüsse und Infektionen mit bestimmten Erregern eine weitere Rolle in der Pathogenese spielen. Epidemiologisch valide Untersuchungen zu Häufigkeit, Verlauf und Prognose der MS im Kindes- und Jugendalter sind bislang weltweit nicht verfügbar. Diese sind jedoch von großer Bedeutung und bilden die Grundlage für die Evaluation der Wirksamkeit von bereits verfügbaren und zukünftig neuen immunmodulatorischen Therapien. Gemeldet werden sollen Kinder und Jugendliche bis zum vollendeten 16 . Lebensjahr, mit der Erstmanifestation einer MS nach Mc-Donald Kri- terien (siehe Monatsschrift Kinderheilkunde, Volume 157, Number 1 / January, 2009). Hauptziel der Studie ist die Inzidenzschätzung der MS im Kindes- und Jugendalter in Deutschland. Daher hoffen wir auf Ihre rege Mitarbeit, um tatsächlich alle Fälle erfassen zu können. Weiterhin sollen die klinischen Symptomatik bei Erstmanifestation und durch eine Langzeiterfassung auch der klinischer Verlauf, Therapiestrategien und die Prognose evaluiert werden.

Studienleitung:

Prof. Dr. med. Jutta Gärtner (Pädiatrie Il, Tel.: 0551-398035, gaertnj@med.uni-goettingen.de), Prof. Dr. med. Wolfgang Brück (Neuropathologie, Tel.: 0551-392700, wbrueck@med.uni-goettingen.de), Universitätsklinikum Göttingen, Robert-Koch-Str. 40, 37075 Göttingen Dr. med. Veit Grote, Pädiatrische Epidemiologie, Institut für Soziale Pädiatrie, Epidemiologie und Jugendmedizin, Universität München, Heiglhofstr. 63, 81377 München, Tel.: 089-71009307, veit.grote@med.uni-muenchen.de

\section{Nicht-bakterielle Osteitis und bakterielle Osteo- myelitis}

Im Januar 2009 wurde die ESPED-Erhebung zur „Nicht-bakterielle Osteitis“ (NBO) um Fälle mit einer Neudiagnose einer bakteriellen Osteomyelitis (BOM) erweitert. Die Diagnose der NBO stellt derzeit eine Herausforderung für alle Kliniker dar, weil die BOM mit ihren therapeutischen Konsequenzen auch heute noch im Bewusstsein der Ärzte eine fest verankerte Erkrankung ist und die Differenzierung zu den wichtigsten Differenzialdiagnosen BOM und Knochentumoren in der Praxis häufig schwierig ist. Die Diagnose „bakterielle Osteomyelitis" wird jedoch vermutlich viel zu häufig gestellt. Patienten, die in Wirklichkeit an einer rheumatischen „Autoinflammations- 
Osteomyelitis“ - der NBO - erkrankt sind, werden somit zu invasiv (d.h. mit operativen Eingriffen) und über viele Wochen antibiotisch behandelt. Dafür werden stationäre Aufenthalte verordnet, die die Kinder, Eltern und das Gesundheitssystem belasten. Populationsbezogene $\mathrm{Da}$ ten sowie Inzidenzdaten zur Osteomyelitis liegen kaum vor. Das deutsche Standardwerk der pädiatrischen Infektiologie (DGPI Handbuch 2009) stellt fest, dass es „keine zuverlässigen Angaben über die Häufigkeit" gibt.Anhand der Studie soll daher zum einen die Inzidenz der Osteomyelitis (bakteriell oder nicht-bakteriell) im Kindesalter genauer ermittelt werden. Zum anderen soll die Validität eines diagnostischen Scores (Jansson et al, Arthritis \& Rheumatism, 2009) zur Differenzierung der NBO von der BOM überprüft werden. Darüber hinaus werden Angaben zur klinischen und laborchemischen Präsentation sowie Angaben zur Therapie erhoben.

\section{Studienleitung:}

Dr. med. Annette Jansson, Oberärztin in der Rheumatologie / Dr. med. Veit Grote, Epidemiologe, Dr. von Haunersches Kinderspital, Lindwurmstr. 4, 80337 München, Tel.: 089-5160-7848, Fax: 089-5160-3964 annette.jansson@ med.uni-muenchen.de; veit.grote@ med.uni-muenchen.de

\section{Personalia}

Die Gesellschaft für Pädiatrische Nephrologie (GPN) zeichnete auf ihrer Jahrestagung, die im März 2009 gemeinsam mit der niederländischen Fachgesellschaft in Amsterdam veranstaltet wurde, herausragende Präsentationen junger Wissenschaftler mit dem Elke-Kröner-Fresenius-Preis aus.

Für ihre Vorträge auf der Tagung wurden ausgezeichnet: Stefanie Jeruschke (Düsseldorf), Dr. Heiko Billing (Heidelberg) und Lea Haisch (Berlin). Posterpreise erhielten Gita Gemulla (Heidelberg), Dr. Markus Benz (München) und Mrs. Renske Raaijmakers (Nijmegen, Niederlande).

Am 15.05.09 hat die Norddeutsche Gesellschaft für Kinder- und Jugendmedizin auf ihrer 58. Jahrestagung in Wolfsburg erneut den Heinrich-FinkelsteinPreis verliehen. Der mit 5.000 Euro dotierte Preis wurde vom Gutachtergremium 2 Bewerbern zu gleichen Teilen zuerkannt: Herrn Dr. med. Georg Bohn aus der Kinderklinik/Pädiatrische Hämatologie und Onkologie der Medizinischen Hochschule Hannover für seine Arbeit „Primary immunodeficiency syndrome caused by deficiency of the endosomal adaptor protein p14" sowie Frau Dr. med. Nele Stahlmann aus der Universitätskinderklinik Lübeck für ihre Arbeit "Outcome of extremely premature infants in a geographically defined region in Germany. Neurosensory, cognitive, behavioral outcome, health-related quality of life".

\section{Personalia}

\section{Herrn Prof. Dr. Heribert Jürgens zum 60. Geburtstag}

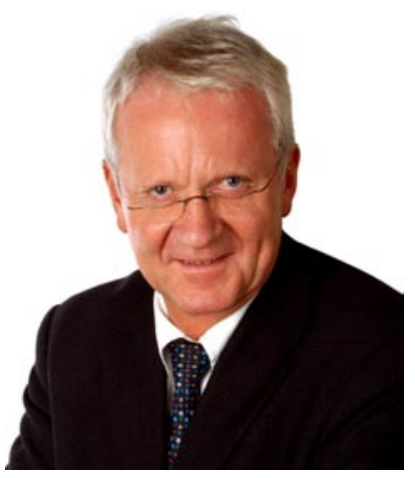

$\triangle$ Professor Dr. Heribert Jürgens
Am 03. Juli 2009 wird Prof. Dr. med. Heribert Jürgens 6o Jahre alt.

Nach seinem Abitur in Bonn studierte Heribert Jürgens Medizin in Düsseldorf und Glasgow und legte sein Staatsexamen 1974 ab. Nach der Medizinalassistentenzeit in Düsseldorf erhielt er 1976 die Approbation und arbeitete zunächst an der Universitätskinderklinik in Düsseldorf, von wo aus er mit einem Stipendium der Deutschen Krebshilfe für ein Jahr zum Memorial Sloan Kettering Cancer Center nach New York wechselte. Ein Jahr nach seiner Facharztanerkennung als Arzt für Kinderheilkunde habilitierte er sich für unser Fachgebiet an der Medizinischen Fakultät der Universität Düsseldorf mit dem Thema „Die hochdosierte Methotrexatbehandlung mit Citrovorumfaktor-Rescue als therapeutisches Konzept" und erhielt die Venia legendi.

1991 wurde er als Direktor der Abteilung Pädiatrische Hämatologie/Onkologie an die Westfälische Wilhelms - Universität Münster berufen. Diese Position hat er bis heute inne. 1980 initiierte er die kooperativen E.W.I.N.G.-Sarkom-Studien in Deutschland und Österreich. Seitdem sind vier große Studien zum Abschluss gekommen: CESS 81, CESS 86, EICESS
92 und E.W.I.N.G. 99. An den Studien CESS 81 und 86 waren die GPOH Kliniken, an der Studie EICESS 92 bereits die Kliniken aus Großbritannien beteiligt. Die Studie E.W.I.N.G. 99 ist ein großes europäisches Konsortium aus allen wichtigen europäischen Arbeitsgruppen. In diese Studie wurden mittlerweile 2671 Patienten aufgenommen. Bemerkenswert ist, dass das Alter der in diesen Studien behandelten Patienten bis zum 50.Lebensjahr ausgedehnt werden konnte. Konsequenterweise leitet $\mathrm{He}$ ribert Jürgens seit 1992 als Intergroup Chairman die Internationalen Cooperativen EWINGSarkom-Studien. Neben der Fortentwicklung der Chemotherapie haben diese Studien einen hohen Standard im Bereich der Lokaltherapie gesetzt. Durch intensive Arbeit im Detail, für die viele hunderte Gespräche mit Operateuren und Strahlentherapeuten notwendig waren, wurde die optimale Kombination aus Operation und Radiotherapie herausgearbeitet. Nicht zuletzt dieser Arbeit ist es zu verdanken, dass Patienten mit Ewingsarkomen heute eine hohe Überlebenschance haben. Zudem hat die Studiengruppe die Rolle der Biologie des Ewingsarkoms und insbesondere der Molekularbiologie bearbeitet. In dieser Funktion ist Heribert Jürgens nicht nur national sondern weltweit sichtbar. Bisweilen kann es passieren, dass er auf Kongressen auch als Dr. Ewing angesprochen wird.

Über die intensive klinische Forschung hinaus, hat er sich in den letzten Jahren in internationalen pädiatrisch onkologischen Gesellschaften engagiert. Seit 1983 ist er Mitglied des Vorstands der Gesellschaft für Pädiatrische Onkologie und Hämatologie (GPOH), dessen Vorsitzender er von 2002-2006 war. Von 19941998 war er Mitglied des Vorstands der International Society of Paediatric Oncology (SIOP). Darüber hinaus hat er sich auch 
in die akademische Selbstverwaltung seiner medizinischen $\mathrm{Fa}$ kultät eingebracht und von 1998 2002 als Prodekan mitgearbeitet und sie von 2002-2006 als Dekan geleitet. Zusätzlich fand er noch Zeit für intensive Drittmitteleinwerbungen und für eine intensive Nachwuchsförderung. Drei seiner ehemaligen Mitarbeiter leiten mittlerweile renommierte Kliniken im In-und Ausland. In seiner Klinik ist Heribert Jürgens als Inkarnation der Interdisziplinarität bekannt. Die lokaltherapeutischen Fallkonferenzen seiner Abteilung schließen sämtliche Subdisziplinen ein und dienen jungen Assistenten und Studierenden als Lehrstunde, Fachärzten und reiferen Assistenzärzten als Erprobungsfeld der eigenen Einschätzungen und erfahrenen Oberärzten als Dis-

kussionsforum auf höchstem Niveau. Unvergessen sind und bleiben die Abende am Rande verschiedenster weltweiter Tagungen mit Heribert Jürgens. Hier zeigt sich natürlich die rheinische Frohnatur aber gleichzeitig der höchste Respekt vor Menschen aller Schichten.

Die Deutsche Gesellschaft für Kinder- und Jugendmedizin und die Gesellschaft für Pädiatrische Onkologie und Hämatologie gratulieren Herrn Prof. Jürgens herzlich zu seinem Geburtstag und wünschen ihm weiterhin viel Erfolg im Dienst der Pädiatrischen Onkologie sowie viele weitere Jahre voller Freude und in guter Gesundheit.

Prof. Dr. med. Thomas Klingebiel

\section{Kongresskalender}

2009
Mannheim, 03.09.-06.09.2009
105. Jahrestagung der Deutschen
Gesellschaft für Kinder- und
Jugendmedizin (DGKJ)
Leitung: Prof. Dr. HJ. Böhles
Tel. 030/308 777-90, Fax-999
info@dgkj.de
www.dgkj2009.de

Köln, 23.09.-26.09.2009

19. Jahrestagung der Gesellschaft für Kinder- und Jugendrheumatologie (GKJR)

Leitung: Prof. Dr. G. Horneff www.gkjr.de

Hamburg, 24.09.-26.09.2009 46. Jahrestagung der Gesellschaft für Pädiatrische Radiologie Leitung: Prof. Dr. K. Helmke www.kinderradiologiekongress2009. de
Berlin, 24.10.-28.10.2009

9. Weltkongress für Perinatale Medizin

Leitung: Prof. Dr. J. Dudenhausen www.wcpm9.org

Kiel, 13.11.-15.11.2009

4. Gemeinsamer Kongress der Arbeitsgemeinschaften für Pädiatrische Endokrinologie und für Pädiatrische Diabetologie Leitung: Prof. Dr. P.-M. Holterhus, Dr. B. Heidtmann

www.ape-agpd2009.de

Hünfeld, 19.11.-21.11.2009

5. Jahrestagung der Gesellschaft für Pädiatrische Psychosomatik Leitung: G. Bürk

www.agpps-ev.de

Frankfurt/Main, 20.11.-21.11.2009 74. Wissenschaftliche Halbjahrestagung der Gesellschaft für Pädiatrische Hämatologie und Onkologie (GPOH)

Leitung: Prof. Dr. T. Klingebiel

www.kinderkrebsinfo.de

Florenz, 03.12.-06.12.2009

Excellence in Paediatrics

Leitung: Prof. Dr. A. Rubino, S. Logan

www.excellence-in-paediatrics.org
2010

Hamburg, 25.03.-27.03.2010

41. Jahrestagung der Gesellschaft für Pädiatrische Nephrologie Leitung: Prof. Dr. D. E. Müller-Wiefel www.gpn2010.de

München, 06.05.-08.05.2010 „The Power of Programming - International Congress on Developmental Origins of Health and Disease"

Leitung: Prof. Dr. B. Koletzko www.metabolic-programming.org/ munich2010

Heidelberg, 07.05.-08.05.2010 Entwicklungen und Perspektiven der Kinder- und Jugendmedizin 150 Jahre Pädiatrie in Heidelberg Leitung: Prof. Dr. Prof. Dr. h. c. G. F. Hoffmann www.klinikum.uni-heidelberg.de/ kinder

Hier erscheinen nur die Ankündigungen für wissenschaftliche Kongresse. Die Terminankündigungen für Weiterund Fortbildungsmaßnahmen entnehmen Sie bitte der Homepage der DGKJ: www.dgkj.de/terminkalender.html schen Gesellschaft für Kinder-

und Jugendheilkunde

Leitung: Univ.-Prof. Dr. W. Müller

Tel. +433163854099

Fax +433163853300

congress.graz@mcg.at

www.medunigraz.at/kinderklinik/

congress2009

Weimar, 03.10.-06.10.2009

41. Jahrestagung der Deutschen Gesellschaft für Pädiatrische

\section{Kardiologie}

Leitung: Prof. Dr. J. Hess

www.kinderkardiologie.org

Hamburg, 09.10.-12.10.2009

50th Annual Meeting of the

European Society for Paediatric Research

Leitung: Prof. Dr. H. v. Goudoever

Tel. +41 229080488

Fax +41227322850

Esprog@kenes.com

www.kenes.com/paediatric-research

\section{Geschäftsstelle der DGKJ}

Wir sind für Sie wie folgt erreichbar:

Chausseestr. 128/129, D-10115 Berlin

Tel.: +49/30/30877 79-0 - Fax: +49/30/30877 79-99

E-Mail:info@dgkj.de·Internet: www.dgkj.de

Geschäftsstelle der ÖGKJ für Öffentlichkeitsarbeit und Standesführung

Wir sind für Sie wie folgt erreichbar:

Mag. Claudia Fabisch

Universitätsklinik für Kinder- und Jugendheilkunde Graz

Auenbruggerplatz 30, A-8036 Graz

Tel.: +43 (0) 3163852061 - Fax: + $43(0) 3163853300$

E-Mail: claudia.fabisch@klinikum-graz.at

Internet: www.docs4you.at

www.dgkj.de

www.docs4you.at

Unter diesen Adressen sind die Deutsche Gesellschaft für Kinder- und Jugendmedizin und die Österreichische Gesellschaft für Kinder- und Jugendheilkunde für Sie im Internet erreichbar. 


\section{Geburtstage}

Die Deutsche Gesellschaft für Kinder- und Jugendmedizin und die Österreichische Gesellschaft für Kinder- und Jugendheilkunde gratulieren herzlich ihren Mitgliedern, die im nächsten Monat einen „besonderen“ Geburtstag feiern:

\section{Jahre und älter werden:}

01.08 .1920

Herr Prof. Dr. Walter Marget, Starnberg

03.08.1920

Herr Dr. Horst Chomse, Soest

04.08.1920

Herr Dr. R. Würkert, Schramberg

04.08.1924

Frau Dr. Luise Pache, Grünwald

04.08.1927

Herr Dr. Detlev Hosenfeld, Kiel

05.08.1920

Herr Prof. Dr. Heinz Singer,

Oberaudorf

05.08.1928

Frau Dr. Lotte Anke, Hagen

05.08.1929

Herr Dr. H. Zoepffel, Würzburg

06.08.1921

Frau Dr. Irmgard Trösch,

Tauberbischofsheim

07.08.1920

Herr Dr. Dieter Scheffzek, Heidelberg

07.08.1921

Frau Dr. Lieselotte Reichenbach,

Spaichingen

07.08.1922

Herr Dr. Werner Gladel, Saarlouis

08.08.1922

Frau Dr. Gisela Biermann, Köln

08.08.1922

Frau Dr. Anneliese Czapski, Weimar

08.08.1922

Herr Prof. Dr. Hermann Gutheil,

Erlangen

09.08.1928

Herr Dr. Wolfgang Anke, Hagen

10.08.1921

Frau Dr. Gerda Bitterolf, München

11.08.1919

Frau Dr. Rita Blume, Unna

11.08.1924

Herr Dr. Hans-J. Krug, Essen

12.08.1920

Frau Dr. Hildegard Schmidt,

Wiesbaden

13.08.1919

Herr Dr. Heinz Kohler, Grönenbach

13.08.1922

Frau Dr. Hildegard Neumann,

Hamburg

13.08.1928

Herr Dr. Hans Wolfgang Schmidt,

Pöring

\subsubsection{1}

Frau Dr. Lore Lieb-Zürn, Plochingen

14.08.1925

Herr Prof. Dr. Gebhard Flatz, Bonn

15.08.1929

Frau Dr. Maria Boulanger-Raida, Bonn

17.08.1928

Herr Dr. Otto Heinrich Klöß, Frankfurt

18.08.1926

Frau Dr. Helga Sommer, Wiesbaden

21.08.1929

Frau Dr. Edeltraud von Eicken, Essen

22.08.1920

Frau Dr. Gertrud Lange,

Bad Neuenahr-Ahrweiler

22.08.1927

Frau Dr. Angela Cordier, Aachen

23.08.1919

Frau Dr. I. Ebert-Schmiedel, Stuttgart

23.08.1923

Herr Dr. Günther Preusche, Sankt

Augustin

23.08.1924

Herr Dr. Rudolf Kaulen, Neuss

24.08.1924

Herr Dr. Josef Diwisch, Hamm

24.08.1925

Herr Dr. Günter Eilers, Berlin

24.08.1927

Herr Dr. Ulrich Diesing, Kirkel

26.08.1922

Herr Dr. Richard Bormann, Zwickau

28.08.1928

Herr Dr. Oskar Wolff, Essen

31.08.1920

Herr Dr. Hubertus Schulte, Essen

31.08.1926

Herr Dr. Harald Stein, Ludwigshafen

\section{Jahre werden:}

Herr Dr. Jaroslav Cermak, Viersen

04.08.1934

Herr Dr. Klaus Schönfelder, Berlin

07.08.1934

Frau Dr. Elisabeth Happe, Rheine

10.08.1934

Herr Prof. Dr. Helmut Bartels,

Würzburg

10.08.1934

Frau Dr. Evelyn Fenske, Berlin

11.08.1934

Herr Dr. Raul Chavez, Alfter
01.08.1934
17.08.1934

Herr Dr. Dieter Keller, Dresden

19.08.1934

Herr Prof. Dr. Jan-Diether Murken,

München

29.08.1934

Frau Dr. Ursula Kupferschmidt, Berlin

70 Jahre werden:

04.08.1939

Herr Dr. Morad-Reza Ameri, Essen

11.08.1939

Herr Prof. Dr. Hans Georg Schlack, Bonn

13.08.1939

Herr Prof.Dr.med.habil Stefan Wässer,

Geesthacht

14.08.1939

Herr Dr. Arnold Breuer, Solingen

21.08.1939

Herr Carl-Ernst Pilars de Pilar,

Gräfelfing

24.08.1939

Herr Dr. Sami Habash, Cham

26.08.1939

Frau Dr. Ursula John-Grafe,

Steinbach am Wald

\section{Jahre werden:}

01.08.1944

Herr Dr. Jörg Till, Brahmenau

04.08.1944

Herr Dr. Sulaiman Kassir, Berlin

14.08.1944

Frau Dr. Gudrun Waldner, A-Wien

18.08.1944

Herr Dr. Herbert Kerschbaum, A-Horn

Sollte ein Mitglied nicht wünschen, dass sein Geburtstag in dieser Gratulationsliste erwähnt wird, bitten wir um rechtzeitige Nachricht (4 Monate vor dem Geburtstag) an die Deutsche Gesellschaft für Kinder- und Jugendmedizin e. V.,

Chausseestr. 128/129, D-10115 Berlin, info@dgkj.de.
23.08.1944

Herr Dr. Bernd Van Husen, Bochum

29.08.1944

Herr Dr. Wolfgang Peuckert, Freiburg

31.08.1944

Herr Univ.-Prof.Dr. Wilhelm D. Müller,

A-Graz

\section{Jahre werden:}

04.08.1949

Frau Dr. Ulrike Beyer, Neukirchen-Vluyn

09.08.1949

Frau Dr. Dagmar Schütte,

Braunschweig

09.08.1949

Frau Heidrun Tischer, Leuben

11.08.1949

Herr Dr. Josef Althaus, Lübeck

11.08.1949

Herr Dr. Christian Holzleitner,

A-Innsbruck

15.08.1949

Frau Dr. Lena Heijbel, A-Innsbruck

20.08.1949

Herr Prof.Dr.Dr. Werner Blum, Usingen

20.08.1949

Frau Dr. Elisabeth Knolle, Fernwald

24.08.1949

Frau Dipl.-Med. Sabine Berndt,

Nordhausen

26.08.1949

Frau Renate Grützner, Wremen

26.08.1949

Herr Dr. Alexander von Stülpnagel,

Krefeld 
DEUTSCHE GESELLSCHAFT FÜR KINDER- UND JUGENDMEDIZIN e.V.

DGKJ e. V. Geschäftsstelle

Chausseestr. 128/129, D- 10115 Berlin

Fax: +49 (0) $30 / 30877$ 79-99

Aufnahmeantrag

Beze honatsschrift

Beziehen Sie Ihre Monatsschift
doch über eine Mitgliedschaft in der DGKJ!

Hiermit erkläre ich meinen Beitritt zur Deutschen Gesellschaft für Kinderund Jugendmedizin e. V. und bestätige, dass ich approbierte/r Ärztin/Arzt bin.

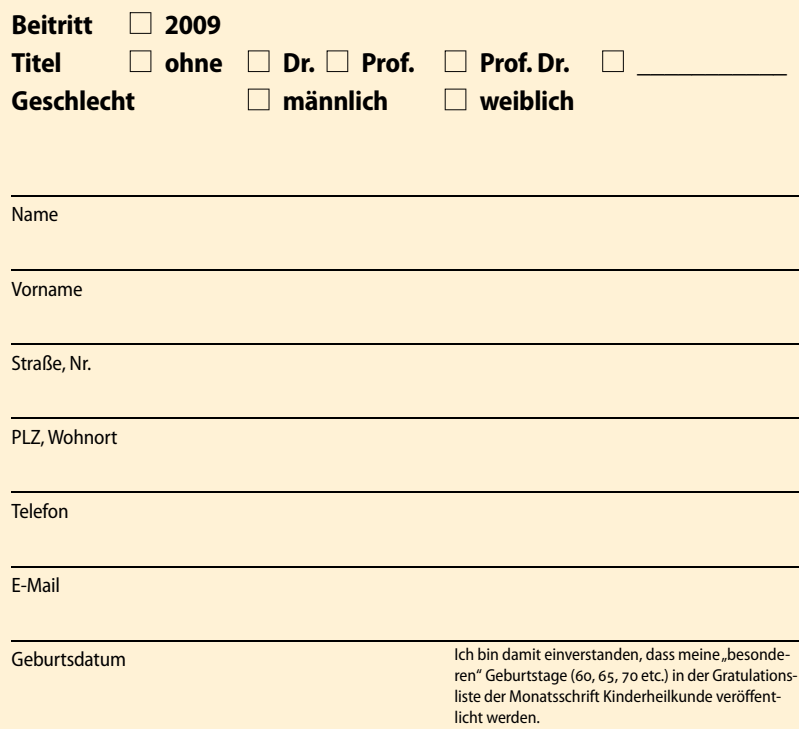

Zutreffend für mich ist folgender Mitgliedsbeitrag pro Kalenderjahr:

$\square$ selbständig oder mit Einnahmen aus Privatliquidationen 107,- $€$

$\square$ nicht selbständig ohne Einnahmen aus Privatliquidationen $87,-€$

$\square$ für Mitglieder in den neuen Bundesländern 67,-€

$\square$ Assistenzärzte im 1. (Berufs-) Jahr 47,- $€$ im 1. Kalenderjahr der Mitgliedschaft

$\square$ Eltern in Elternzeit 47,- $€$ (mit Moki) bzw. beitragsfrei Die Mitgliedschaft schließt den Bezug der Monatsschrift Kinderheilkunde ab Jahresbeginn ein. Die Kosten sind im Beitrag enthalten. Der Mitgliedsbeitrag soll bei Fälligkeit eingezogen werden zu Lasten meines

Kontos-Nr.

$\overline{B 17}$

Bank

Diese Einzugsermächtigung ist jederzeit widerruflich. Sollte das Konto die erforderliche Deckung nicht aufweisen, besteht seitens des kontoführenden Kreditinstitutes keine Verpflichtung zur Einlösung.

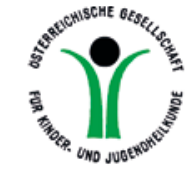

\section{ÖSTERREICHISCHE GESELLSCHAFT} FÜR KINDER- UND JUGENDHEILKUNDE

\section{ÖGKJ \\ Geschäftsstelle für Öffentlichkeitsarbeit und Standesführung}

Mag. Claudia Fabisch

Universitätsklinik für Kinder- und Jugendheilkunde Graz

Auenbruggerplatz 30

A-8036 Graz

Tel.: +43 (0)316 385 2061, Fax: +43 (0)3163853300

E-Mail: claudia.fabisch@klinikum-graz.at

Internet: www.docs4you.at

\section{Mitglieds-Anmeldung}

Beziehen Sie Ihre Monatsschrift doch uber eine Mitgliedschaft in der ÖGKJ!

Familienname

Vorname

Akad. Titel

Krankenhaus/Dienstanschrift oder Privatadresse

\section{Straße, Nr.}

PLZ, Wohnort

Telefon / Fax

E-Mail

Geburtsdatum

Geburtsort

Nationalität

Ort \& Datum der Promotion

Seit wann \& wo in pädiatrischer Facharztausbildung

Facharzt/Fachärztin für Kinder- und Jugendheilkunde seit

Derzeitige berufliche Tätigkeit 